\title{
UNIFORMLY REFLEXIVE STRUCTURES: ON THE NATURE OF GÖDELIZATIONS AND RELATIVE COMPUTABILITY
}

BY

\author{
ERIC G. WAGNER
}

\begin{abstract}
In this paper we present an axiomatic theory within which much of the theory of computability can be developed in an abstract manner. The paper is based on the axiomatically defined concept of a Uniformly Reflexive Structure (U.R.S.). The axioms are chosen so as to capture what we view to be the essential properties of a "gödelization" of a set of functions on arbitrary infinite domain. It can be shown that (with a "standard gödelization") both the partial recursive functions and the meta-recursive functions satisfy the axioms of U.R.S. In the first part of this paper, we define U.R.S. and develop the basic working theorems of the subject (e.g., analogues of the Kleene recursion theorems). The greater part of the paper is concerned with applying these basic results to (1) investigating the properties of gödelizations, and (2) developing an intrinsic theory of relative computability. The notion of relative computability which we develop is equivalent to Turing reducibility when applied to the partial recursive functions. Applied to appropriate U.R.S. on arbitrary domains, it provides an upper-semi-lattice ordering on the set of all functions (both total and partial) on that domain.
\end{abstract}

0. Introduction. In this paper we present the axioms of an abstract theory of computability (the theory of Uniformly Reflexive Structures) and employ it to establish a number of general results on gödelizations and to develop an intrinsic approach to the study of relative computability.

To a first approximation, a Uniformly Reflexive Structure (a U.R.S.) is a set of functions on an arbitrary infinite domain together with a special indexing of the functions by elements of the domain. We call these indexings gödelizations; this terminology is apt in that the partial recursive functions with a "standard gödelization" (such as given by Davis [1]) form a U.R.S.

For each element $u$ of the domain, the indexing gives us a 1-ary function denoted $[u]_{1}$; following Schoenfinkel [6], we extend the indexing, by iteration, to $n$-ary functions $[u]_{n}$ for all positive integers $n$ and elements $u$ of the domain. The requirements for an indexing to be called a gödelization are, again approximately, that, (1) there be a function in the set such that applied to the gödel numbers (indices) $f$ and $g$ of any two functions $[f]_{1}$ and $[g]_{1}$, it gives us a gödel number of their "blend", that is, the function which, for all $x$, equals $\left[[f]_{1}(x)\right]\left([g]_{1}(x)\right) ;(2)$ there be

Received by the editors May 23, 1966 and, in revised form, January 30, 1968. 
a function in the set which, when applied to any three elements $a, b$, and $c$ of the domain, gives us a gödel number of the function which applied to any element $x$ of the domain takes the value $a$ or $b$ according as whether or not $x=c$.

In $\S 1$ we restate these requirements as formal axioms and give an additional axiom-of-convenience for dealing with partial functions. At the end of this section we briefly discuss models for these axioms. In $\$ 2$ we establish some preliminary results and additional necessary notation. These results comprise the fundamental lemmas of the subject.

In $\S 3$ we prove a number of results on gödelizations. These results, while perhaps not of particular interest to recursive function theorists, encompass the results of recursion theory which we have found to be of greatest interest in application in "computer science". We prove that we cannot gödelize a set of total functions; we present two simple normal form theorems for U.R.S. and we prove that gödelizations are infinite-to-one. We show that if two gödelizations index the same set of 1 -ary functions, then they must index the same sets of $n$-ary functions for all positive $n$. We prove that given two gödelizations of a set of functions, there must exist functions in the set which carry one gödelization to the other, and vice versa; that is, that with respect to the first gödelization, there will exist a function $[f]_{1}$ such that, for all elements $u$ of the domain, $\left[[f]_{1}(u)\right]_{1}$ will be the 1-ary function corresponding to $u$ under the second gödelization. Finally, we prove a converse to the last result to the effect that if $[g]_{1}$ and $[f]_{1}$ are total functions such that, for every $u$ and $x$ in the domain, $[f]_{2}\left([g]_{1}(u), x\right)=[u]_{1}(x)$, then the map which carries each element $v$ of the domain to the mapping $\left[[f]_{1}(v)\right]_{1}$ is a gödelization.

The second half of the paper is directed toward the development of an intrinsic theory of relative computability. $\$ 4$ consists of some additional preliminaries. We introduce generalizations of the concepts of computable and semicomputable sets and we extend the operations of primitive recursion and minimalization to arbitrary U.R.S. We begin $\$ 5$ with a means for "constructing" U.R.S. on arbitrary infinite domains. After employing this result to give a particularly simple characterization of the partial recursive functions as a U.R.S., we turn to the study of U.R.S. within which it is possible to "carry out" this "construction". We introduce a special class of U.R.S., the highly constructible U.R.S., which are "self-constructing" using only total functions. We show that the partial recursive functions form a highly constructible U.R.S. and that such U.R.S. exists on all infinite domains. We establish a direct generalization of the Kleene Normal Form Theorem for highly constructible U.R.S. and we employ it to prove that such U.R.S. contain "recursively inseparable" semicomputable sets. In $\$ 6$ we introduce the promised intrinsic definition of relative computability with respect to highly constructible U.R.S. We say of two U.R.S. $I$ and $I^{\prime}$ that $I^{\prime}$ is an extension of $I$ if the set of functions $F\left(I^{\prime}\right)$ associated with $I^{\prime}$ contains the set of functions $F(I)$ associated with $I$. We show that if $I$ is a highly constructible U.R.S. and $f$ is any function (on the same domain) then there exists a U.R.S. $I_{f}$ which is the minimal extension of $I$ which 
contains $f$, i.e., $F(I) \cup\{f\} \subseteq F\left(I_{f}\right)$ and for every U.R.S. $I^{\prime \prime}$, if $F(I) \cup\{f\} \subseteq F\left(I^{\prime \prime}\right)$, then $F\left(I_{f}\right) \subseteq F\left(I^{\prime \prime}\right)$. Conversely, we show that if $I$ is highly constructible, and $I^{\prime}$ is an extension of $I$ (but not necessarily highly constructible) then there exists a function $f$ such that, where $I_{f}$ is the minimal extension of $I$ containing $f$, we have $F\left(I_{f}\right)=F\left(I^{\prime}\right)$. Applying these results to "the" U.R.S. $I_{R}$ of partial recursive functions, we show that for any total function $f$, the set of functions $F\left(I_{f}\right)$ of the minimal extension $I_{f}$ is precisely the set of functions recursive in $f$. On the other hand, where $I$ is highly constructible and $f$ is total, it follows that $I_{f}$ is highly constructible. Thus, the notion of relative computability reduces directly to the natural, and intrinsic, ordering on the highly constructible extensions of a highly constructible U.R.S. To complete the picture, we show that where $f$ is not total, the minimal $f$ extension need not be highly constructible, and that the set of all extensions of a highly constructible U.R.S. form an upper semilattice.

1. Axioms for uniformly reflexive structures. We shall first give the required definitions and axioms and will then motivate and interpret them in terms of the partial recursive functions.

A Uniformly Reflexive Structure (a U.R.S.) $I$ is a pair $I=\langle U, G\rangle$ where $U$ is an arbitrary infinite set and $G: U \rightarrow U^{U}$. (Rather than assume $U$ infinite, it suffices, as can be shown from the results of the next section, to assume that $U$ contains at least three distinct elements.) The set $U$ is called the domain of the U.R.S., the map $G$ is called the gödelization (of the 1-ary functions) of the U.R.S. For all elements $u, x \in U$, we write $[u]_{1}$ for $G(u)$, and $[u]_{1}(x)$ for the value $(G(u))(x)$ of $[u]_{1}$ at $x$. The set $G(U)$, also denoted $F(1, I)$, is called the set of 1-ary functions of $I$. Following Schoenfinkel [6], we extend the gödelization to functions $[u]_{n}: U^{n} \rightarrow U$, of $n$ variables, for all $n>1$ by iteration; namely, for each $u \in U$ and each integer $n>1$, $[u]_{n}$ is defined to be such that, for each $n$-tuple

$x^{(n)}=\left\langle x_{1}, \ldots, x_{n-1}, x_{n}\right\rangle \in U^{n},[u]_{n}\left(x_{1}, \ldots, x_{n-1}, x_{n}\right)=\left[[u]_{n-1}\left(x_{1}, \ldots, x_{n-1}\right)\right]\left(x_{n}\right)$.

For each $n$, the set $\left\{[u]_{n} \mid u \in U\right\}$, of the $n$-ary functions of $I$, is denoted by $F(n, I)$. The set $F(I)=\bigcup_{n=1}^{\infty} F(n, I)$ is called the function set of $I$. When there is no ambiguity we shall omit the subscripts on the functions.

For $I=\langle U, G\rangle$ to be a U.R.S., we require that it satisfy the following axioms:

Axıом 1. There exists $* \in U$ such that, for every $u \in U$,

$$
[u](*)=*=[*](u)
$$

We shall find it convenient, in what follows, to write $V$ for $U-\{*\}$.

Axiom 2. There exists $\alpha \in U$ such that, for all $f, g$, and $x$ in $V$,

(i) $[\alpha](f, g) \neq *$,

(ii) $[[\alpha](f, g)](x)=[f](x,[g](x))=[[f](x)]([g](x))$.

(Note the two expressions on the right-hand side are equal by virtue of our definition of $n$-ary function.) 
Axiom 3. There exists $\psi \in U$ such that, for all $a, b, c$, and $x$ in $V$,

$$
\begin{aligned}
{[[\psi](c, b, a)](x) } & =a & & \text { if } x=c \\
& =b & & \text { if } x \neq c .
\end{aligned}
$$

This completes the statement of the axioms.

If we interpret these axioms in terms of the partial recursive functions, as given say in Davis [1], then the domain $U(=V \cup\{*\})$ is the nonnegative integers plus *, where $*$ corresponds to "undefined". That is, rather than have a partial function undefined for a given argument, we adopt the convention that it take the value $*$ for the argument. For the gödelization $G$ in this case, we can take any gödelization of the 1-ary partial recursive functions. For example, we might take the "standard gödelization" given by Davis so that for every $e, x \in V$,

$$
[e](x)=U\left(\min _{y} T(e, x, y)\right)
$$

where $U$ and $T$ are as in Davis's Theorem 2.1, page 63 of [1]. It follows from the Kleene Iteration Theorem that all the $n$-ary partial recursive functions can be produced from this gödelization by means of iteration.

Axiom 1 may then be interpreted as saying that the result is undefined $(=*)$ when we either apply a function to an undefined argument, or when we apply the function corresponding to an undefined gödel number to any argument. In keeping with this interpretation, we say a function $[u]_{n} \in F(I)$ for any U.R.S. $I$, is $*$-total if it never assumes the value $*$ on $V^{n}$. We shall write $F^{v}(I)$ to denote the set of all *-total functions in $F(I)$.

Axiom 2 provides a uniform means for forming the special composite of two functions which we have called their "blend". The purpose of this axiom, as with Schoenfinkel's Verschmelzungsfunktion [6] (similar to our $[\alpha]_{3}$ ), is that it allows us to establish the closure of $F(I)$ under composition. The "blend" is chosen over the simple composite $[f] \circ[g]$ for the pragmatic reason that it does not appear possible to prove general closure under composition from a similar axiom for the simple composite.

Axiom 3 provides a uniform means for generating (gödel numbers for) all functions which are constant on all but at most one element of $V$. The primary motivation behind this axiom is to establish a basis from which to build up definition-by-cases and predicates.

That Axioms 2 and 3 hold for the partial recursive functions under the "standard gödelization" is easy, but tedious, to show using the Kleene Normal Form and Iteration Theorems plus some elementary constructions. We leave the proof to the reader. Another proof, using a "simpler" gödelization is given in $\$ 5$. In the remainder of this paper we generally abuse terminology by referring to "the U.R.S. of partial recursive functions" when we mean a U.R.S. $I$ whose function set $F(I)$ (with $*$ interpreted as "undefined") is the set of partial recursive functions. 
In both Axioms 2 and 3, we have the situation that the axiom reflects a property of $F(I)$, or more specifically, of the partial recursive functions, in a uniform manner. Axiom 2 reflects the property of closure under "blend", Axiom 3 the property of containing all functions constant on all but at most one element of the domain (other than $*$ ). It is this uniform reflecting of properties that we believe is the essential kernel of the intuitive concept of a gödelization, and it is from it that we derive most of our results and, of course, the name "U.R.S."

As regards models of these axioms other than the partial recursive functions, we show in $\$ 5$ that for all functions $f: N \rightarrow N$ ( $N=$ nonnegative integers), the set of functions recursive in $f$ form the function set of a U.R.S. A more detailed proof is given in [10], and in [11] it is also shown that the partial recursive functions "are the smallest U.R.S. on the nonnegative integers which contains the standard successor function $s(x)=x+1$ ". We also show in $\$ 5$ that U.R.S. can be "constructed" on any infinite domain (this also follows from restating the axioms within the first order predicate calculus and applying Henkin's theorem). It has been shown by Strong [8] that the meta-recursive functions form a U.R.S. and that there exist U.R.S. whose function sets are proper subsets of the set of partial recursive functions.

As a final example, we shall sketch a proof that the (partial) $\Pi_{1}^{1}$-functions form a U.R.S. (see Rogers [5] for underlying concepts and notation). As is well known, for each $n>0$ there exists a recursive relation $T_{n}$ such that an $n$-ary function is a (partial) $\Pi_{1}^{1}$-function if and only if there exists an integer $z$ such that the graph of the function is precisely the set,

$$
\left\{\left\langle x_{1}, \ldots, x_{n}, y\right\rangle \ni(\forall f)(\exists w) T_{n}\left(z, \bar{f}(w),\left\langle x_{1}, \ldots, x_{n}, y\right\rangle\right)\right\} .
$$

We claim that the desired U.R.S. is precisely the U.R.S. $I=\langle G, N \cup\{*\}\rangle$ where $*$ corresponds to "undefined" and $G$ is the indexing given by $T_{1}$; i.e., for all $x, y, z \in N$,

$$
(G(z))(x)=[z](x)=y \Leftrightarrow(\forall f)(\exists w) T_{1}(z, \bar{f}(w),\langle x, y\rangle)
$$

and otherwise, $(G(z))(x)=[z](x)=*$.

It is easily seen that the $n$-ary $\Pi_{1}^{1}$-functions are precisely the functions that arise from the iteration of these 1-ary functions and that these functions satisfy an $S-m-n$ theorem.

The satisfaction of Axiom 1 follows immediately from the definition of $*$.

The existence of the desired function $[\alpha]_{3}$ for Axiom 2 follows from the observation that its graph is precisely the set

$$
\begin{array}{r}
\left\{\langle p , q , x , y \rangle \ni ( \exists y ^ { \prime } ) ( \exists y ^ { \prime \prime } ) \left[(\forall f)(\exists w) T_{1}\left(p, \bar{f}(w),\left\langle x, y^{\prime}\right\rangle\right) \wedge(\forall f)(\exists w) T_{1}\left(q, \bar{f}(w),\left\langle x, y^{\prime \prime}\right\rangle\right)\right.\right. \\
\left.\left.\wedge(\forall f)(\exists w) T_{1}\left(y^{\prime}, f(w),\left\langle y^{\prime \prime}, y\right\rangle\right)\right]\right\} .
\end{array}
$$

This set is clearly $\Pi_{1}^{1}$ (see Rogers, Theorem 16,1, III [5]), and by the $S-m-n$ theorem, we can pick $\alpha$ such that $[\alpha]_{2}$ is total as required. 
The satisfaction of Axiom 3 follows from the fact that the desired function $[\psi]_{3}$ is clearly recursive and thus $\Pi_{1}^{1}$.

The above result, and similar results, can also be conveniently proved using results due to Strong [9].

2. Preliminary results. The following results are presented in order to establish notation and to make the paper self-contained. For a more detailed presentation, see [10]. To give a brief illustration of the nature of the complete proofs, we have given the proof of $2.1(\mathrm{~d})$ in detail.

THEOREM 2.1. In every U.R.S. $I=\langle U, G\rangle$, there exists:

(a) for each $x \in V$, a constant function $\left[k_{x}\right]_{1}$ such that, for all $y \in V,\left[k_{x}\right](y)=x$;

(b) an identity function $[i]_{1}$ such that, for all $x \in V,[i](x)=x$;

(c) a function $[\beta]_{1}$ such that, for all $x, y \in V,[[\beta](x)](y)=x$, indeed $[\beta](x)=k_{x}$;

(d) a function $[\gamma]_{2}$ such that, for all $f, g$, and $x$ in $V,[[\gamma](f, g)](x)=[f]([g](x))$, i.e., $F(1, I)$ is closed under simple composition.

Proof. It suffices to give specific gödel numbers for the desired functions as follows:

(a) take $k_{x}=[\psi](x, x, x)$;

(b) where $c$ is some fixed element of $V$, take $i=[\alpha]\left([\psi](c, c), k_{c}\right)$;

(c) take $\beta=[\alpha]([\alpha](\psi, i), i)$;

(d) we give the derivation of $\gamma$ in detail. Let $f, g$, and $x$ be elements of $V$. Then

$$
\begin{aligned}
{[f]([g](x)) } & =\left[\left[k_{f}\right](x)\right]([g](x)) \\
& =\left[[\alpha]\left(k_{f}, g\right)\right](x) \\
& =[[\alpha]([\beta](f), g)](x) \\
& =[[\alpha]([\beta](f))](g, x) \\
& =\left[\left[\left[k_{\alpha}\right](f)\right]([\beta](f))\right](g, x) \\
& =\left[[\alpha]\left(k_{\alpha}, \beta\right)\right](f, g, x)
\end{aligned}
$$

Thus, it suffices to take $\gamma=[\alpha]\left(k_{\alpha}, \beta\right)=[\alpha]([\beta](\alpha), \beta)$.

THEOREM 2.2. In the function set of every U.R.S. $I=\langle U, G\rangle$, there exists for each integer $n>0$

(a) a function $\left[\gamma_{n}\right]_{2}$ such that, for all $f, g \in V$ and $x^{(n)} \in V^{n},\left[\left[\gamma_{n}\right](f, g)\right]\left(x^{(n)}\right)$ $=[f]\left([g]\left(x^{(n)}\right)\right)$;

(b) a function $\left[\alpha_{n}\right]_{2}$ such that, for all $f, g \in V$ and $x^{(n)} \in V^{n},\left[\left[\alpha_{n}\right](f, g)\right]\left(x^{(n)}\right)$ $=[f]\left(x^{(n)},[g]\left(x^{(n)}\right)\right)$;

(c) a function $\left[\beta_{n}\right]_{n}$ such that, for all $y, z \in V$ and $x^{(n-1)} \in V^{n-1},\left[\left[\beta_{n}\right]\left(y, x^{(n-1)}\right)\right](z)$ $=y$;

(d) a function $\left[u_{n, j}\right]_{n}$ for each $j, 1 \leqq j \leqq n$ such that, for any $\left\langle x_{1}, \ldots, x_{j}, \ldots, x_{n}\right\rangle \in V^{n}$, $\left[u_{n, j}\right]\left(x_{1}, \ldots, x_{j}, \ldots, x_{n}\right)=x_{j}$. 
Proof. (a) take $\gamma_{1}=\gamma$ and, for $n \geqq 1$, take $\gamma_{n+1}=[\gamma]\left(\gamma, y_{n}\right)$;

(b) take $\alpha_{1}=\alpha$ and, for $n \geqq 1$, take $\alpha_{n+1}=[\gamma]\left(\alpha,[\gamma]\left(\alpha_{n}\right)\right)$;

(c) take $\beta_{1}=\beta$ and, for $n \geqq 1$, take $\beta_{n+1}=\left[\gamma_{n+1}\right]\left(\beta, \beta_{n}\right)$;

(d) define $[\beta]^{0}=[i],[\beta]^{1}=[\beta]$ and, for $n \geqq 1,[\beta]^{n+1}=[\beta] \circ[\beta]^{n}$ (these powers of $[\beta]$ are then in $F(I)$ by virtue of induction using $[\gamma]$ ), then it suffices to take, $u_{n, n}$ $=[\beta]^{n-1}(i)$ and, for $j<n$, take $u_{n, j}=[\beta]^{j-1}\left(\beta_{n-j}\right)$.

Having established the existence of the above functions in an arbitrary U.R.S. $I=\langle U, G\rangle$, we are now in a position to prove that any appropriately well-formed expression formed of elements of $U$, square brackets, parentheses, and variables $x_{1}, x_{2}, x_{3}, \ldots$ corresponds to a function in $F(I)$. This result will allow us to establish the existence of new functions in $F(I)$ without having to display specific gödel numbers.

Let $I=\langle U, G\rangle$ be a U.R.S., let $U$ be called the set of constants of $I$, let $X=\left\{x_{1}, x_{2}, x_{3}, \ldots\right\}$ be a set of variables, $X \cap U=\varnothing$. We define a term (well-formed expression) in $I$ as follows:

1. Any constant or variable is a term.

2. If $t_{0}, t_{1}, t_{2}, \ldots, t_{n}$ are terms, then $\left[t_{0}\right]\left(t_{1}, t_{2}, \ldots, t_{n}\right)$ is a term.

3. All terms are given by 1 and 2 .

Let $T$ denote the set of terms. By an assignment we shall mean a map $a: X \rightarrow U$. An assignment $a$ may be extended to a map $\bar{a}: T \rightarrow U$, as follows:

Let $t$ be a term, then

$$
\begin{aligned}
\bar{a}(t) & =t & & \text { if } t \text { is a constant } \\
& =a(t) & & \text { if } t \text { is a variable. }
\end{aligned}
$$

Lastly, when $t=\left[t_{0}\right]\left(t_{1}, t_{2}, \ldots, t_{n}\right)$,

$$
\bar{a}(t)=\bar{a}\left(\left[t_{0}\right]\left(t_{1}, t_{2}, \ldots, t_{n}\right)\right)=\left[\bar{a}\left(t_{0}\right)\right]_{n}\left(\bar{a}\left(t_{1}\right), \bar{a}\left(t_{2}\right), \ldots, \bar{a}\left(t_{n}\right)\right) .
$$

TheOREM 2.3. Let $I=\langle U, G\rangle$ be a U.R.S. and let $t$ be a term in $I$, then there exists an integer $n$, and a constant $\tau \in U$ such that, for every assignment $a: X \rightarrow U$,

$$
\bar{a}(t)=\bar{a}\left([\tau]\left(x_{1}, x_{2}, \ldots, x_{n}\right)\right)=[\tau]_{n}\left(a\left(x_{1}\right), a\left(x_{2}\right), \ldots, a\left(x_{n}\right)\right) .
$$

Proof. We proceed by induction on the form of $t$. If $t$ is a constant $u \in U$, then take $\tau=k_{u}, n=1\left(k_{*}=*\right)$. If $t$ is a variable $x_{i}$, then take $\tau=u_{i, i}, n=i$. Now say $t=\left[t_{0}\right]\left(t_{1}, t_{2}, \ldots, t_{r}\right)$ where the result holds for $t_{0}, t_{1}, \ldots, t_{r}$. Then, for each assignment $a: X \rightarrow U$,

$$
\begin{aligned}
& \bar{a}(t)=\bar{a}\left(\left[t_{0}\right]\left(t_{1}, t_{2}, \ldots, t_{r}\right)\right)=\left[\bar{a}\left(t_{0}\right)\right]_{r}\left(\bar{a}\left(t_{1}\right), \bar{a}\left(t_{2}\right), \ldots, \bar{a}\left(t_{r}\right)\right) \\
& \text { by the definition of } \bar{a}, \text { and } \\
&=\left[\bar{a}\left(\left[\tau_{0}\right]\left(x_{1}, \ldots, x_{n(0)}\right)\right)\right]_{r}\left(\bar{a}\left(\left[\tau_{1}\right]\left(x_{1}, \ldots, x_{n(1)}\right)\right), \ldots, \bar{a}\left(\left[\tau_{r}\right]\left(x_{1}, \ldots, x_{n(r)}\right)\right)\right)
\end{aligned}
$$

for some $\tau_{0}, \tau_{1}, \ldots, \tau_{r} \in U$ and integers $n(0), n(1), \ldots, n(r)$ by the induction hypothesis. 
Now pick $n$ to be the largest of $n(0)$ through $n(r)$. Then, by the above theorems we have that, for each $0 \leqq i \leqq r$

$$
\begin{aligned}
\bar{a}\left(\left[\tau_{i}\right]\left(x_{1}, \ldots, x_{n(i)}\right)\right) & =\left[\tau_{i}\right]_{n(i)}\left(a\left(x_{1}\right), \ldots, a\left(x_{n(i)}\right)\right) \\
& =\left[\tau_{i}\right]\left(\left[u_{n, 1}\right]\left(a\left(x_{1}\right), \ldots, a\left(x_{n}\right)\right), \ldots,\left[u_{n, n(i)}\right]\left(a\left(x_{1}\right), \ldots, a\left(x_{n}\right)\right)\right) \\
& =\left[\tau_{i}^{\prime}\right]\left(a\left(x_{1}\right), \ldots, a\left(x_{n}\right)\right)
\end{aligned}
$$

where

$$
\tau_{i}^{\prime}=\left[[ \alpha _ { n } ] \left(\ldots\left(\left[\left[\alpha_{n}\right]\left(\left[\left[\alpha_{n}\right]\left(\left[\left[\gamma_{n}\right]\left(\tau_{i}\right)\right]\left(u_{n, 1}\right)\right)\right]\left(u_{n, 3}\right)\right) \ldots\right)\right]\left(u_{n, n(i)}\right) .\right.\right.
$$

Thus, we have

$$
\begin{aligned}
\bar{a}(t) & =\left[\bar{a}\left(\left[\tau_{0}^{\prime}\right]\left(x_{1}, \ldots, x_{n}\right)\right)\right]_{r}\left(\bar{a}\left(\left[\tau_{1}^{\prime}\right]\left(x_{1}, \ldots, x_{n}\right)\right), \ldots, \bar{a}\left(\left[\tau_{r}^{\prime}\right]\left(x_{1}, \ldots, x_{n}\right)\right)\right) \\
& =\left[\bar{a}\left(\left[\tau_{0}^{\prime}\right]\left(x_{1}, \ldots, x_{n}\right)\right]\left(\left[\tau_{1}^{\prime}\right]\left(x_{1}, \ldots, x_{n}\right), \ldots,\left[\tau_{r}^{\prime}\right]\left(x_{1}, \ldots, x_{n}\right)\right)\right) \\
& =\bar{a}\left([\tau]\left(x_{1}, \ldots, x_{n}\right)\right)
\end{aligned}
$$

where

$$
\tau=\left[\alpha_{n}\right]\left(\ldots\left[\alpha_{n}\right]\left(\left[\alpha_{n}\right]\left(\tau_{0}^{\prime}, \tau_{1}^{\prime}\right), \tau_{2}^{\prime}\right), \ldots, \tau_{r}^{\prime}\right)
$$

by 2.2. This proves the result for $t$, and thus, by induction, for all terms.

The following two results show that we can build up functions in a U.R.S. by means of definition-by-cases.

THEOREM 2.4. In every U.R.S. $I=\langle U, G\rangle$

(a) if $u, v, a \in V$, then there exists $[g]_{1} \in F(I)$ such that, for every $x \in V$,

$$
\begin{aligned}
{[g](x) } & =[u](x) \quad \text { if } x \neq a \\
& =[v](x) \text { if } x=a
\end{aligned}
$$

(b) given $n>0$ pairs $\langle a(i), b(i)\rangle$ of elements of $V$ where $b(i) \neq b(j)$ if $i \neq j$, and an element $c \in V$, then there exists $[w]_{1} \in F(I)$ such that, for every $x \in V$,

$$
\begin{aligned}
{[w](x) } & =a(i) & & \text { if } x=b(i) \\
& =c & & \text { if } x \notin\{b(1), \ldots, b(n)\} .
\end{aligned}
$$

Proof. (a) By 2.3 there exists $[g]_{1} \in F(I)$ such that, for all $x \in V,[g](x)=$ $[[\psi](a, u, v, x)](x)$, and this gives the desired result by Axiom 3 .

(b) By 2.4(a) there exists $\left[g_{1}\right]_{1} \in F(I)$ such that, for all $x \in V$,

$$
\begin{aligned}
{\left[g_{1}\right](x) } & =a(1)=\left[k_{a(1)}\right](x) & & \text { if } x=b(1) \\
& =c=\left[k_{c}\right](x) & & \text { if } x \neq b(1) .
\end{aligned}
$$

Then, again using $2.4(\mathrm{a})$, define $\left[g_{i}\right]_{1}$ for $i=2, \ldots, n$ to be such that, for all $x \in V$,

$$
\begin{aligned}
{\left[g_{i}\right](x) } & =a(i)=\left[k_{a(i)}\right](x) & & \text { if } x=b(i) \\
& =\left[g_{i-1}\right](x) & & \text { if } x \neq b(i) .
\end{aligned}
$$

Clearly, then, it suffices to take $[w]_{1}=\left[g_{n}\right]_{1}$. 
THEOREM 2.5. For each pair of integers $\langle n, m\rangle$ and distinct elements $a_{1}, a_{2}, \ldots, a_{n}$ in $V$, there exists $h_{n, m} \in V$ such that, for any $f_{0}, f_{1}, \ldots, f_{n}, x_{1}, \ldots, x_{m} \in V$

$$
\begin{aligned}
& {\left[h_{n, m}\right]\left(f_{0}, f_{1}, \ldots, f_{n}, x_{1}, \ldots, x_{m}\right)=\left[f_{1}\right]\left(x_{1}, \ldots, x_{m}\right) \text { if }\left[f_{0}\right]\left(x_{1}, \ldots, x_{m}\right)=a_{1}} \\
& =\left[f_{2}\right]\left(x_{1}, \ldots, x_{m}\right) \text { if }\left[f_{0}\right]\left(x_{1}, \ldots, x_{m}\right)=a_{2} \\
& =\left[f_{n}\right]\left(x_{1}, \ldots, x_{m}\right) \text { if }\left[f_{0}\right]\left(x_{1}, \ldots, x_{m}\right)=a_{n} .
\end{aligned}
$$

Proof. For the sake of convenience, we shall denote $\left(f_{0}, f_{1}, \ldots, f_{n}, x_{1}, \ldots, x_{m}\right)$ by $\left(f_{0}, f^{(n)}, x^{(m)}\right)$. By 2.4 there exists $[w] \in F(I)$ such that, for all $x \in V$,

$$
\begin{aligned}
{[w](x) } & =f_{i} & & \text { if } x=a_{\imath}, i>0, \\
& =f_{0} & & \text { otherwise. }
\end{aligned}
$$

By 2.2 we have $\left[\beta_{n}\right]\left(f_{0}, f^{(n)}, x^{(m)}\right)=\left[f_{0}\right]\left(x^{(m)}\right)$, and $\left[\left[\beta_{n+1}\right]\left(f_{i}\right)\right]\left(f_{0}, f^{(n)}, x^{(m)}\right)=\left[f_{i}\right]\left(x^{(m)}\right)$. Combining these we get

$$
\begin{aligned}
{\left[\left[\beta_{n+1}\right]\left([w]\left(\left[\beta_{n}\right]\left(f_{0}, f^{(n)}, x^{(m)}\right)\right)\right)\right]\left(f_{0}, f^{(n)}, x^{(m)}\right) } & =f_{i}\left(x^{(m)}\right) & & \text { if }\left[f_{0}\right]\left(x^{(m)}\right)=a_{i}, i>0 \\
& =f_{0}\left(x^{(m)}\right) & & \text { otherwise. }
\end{aligned}
$$

The existence of the desired element $h_{n, m}$ of $V$ then follows immediately by 2.3 .

THEOREM 2.6 (ITERATION THEOREM). For each integer $n>0$, there exists $\left[s_{n}\right]_{n+1}$ $\in F(I)$ such that, for all $f \in V, x^{(n)} \in V^{n}, m>0$ and $y^{(m)} \in V^{m},\left[s_{n}\right]\left(f, x^{(n)}\right) \neq *$ and $\left[\left[s_{n}\right]\left(f, x^{(n)}\right)\right]\left(y^{(m)}\right)=[f]\left(x^{(n)}, y^{(m)}\right)$.

Proof. Consider that

$$
\begin{aligned}
{[f]\left(x^{(n)}, y^{(m)}\right) } & =[f]\left(x_{1}, \ldots, x_{n}, y_{1}, \ldots, y_{m}\right) \\
& =\left[[f]\left(x_{1}, \ldots, x_{n}, y_{1}\right)\right]\left(y_{2}, \ldots, y_{m}\right) \\
& =\left[\left[[\beta]\left(f, y_{1}\right)\right]\left([\beta]\left(x_{1}, y_{1}\right)\right), \ldots,[\beta]\left(x_{n}, y_{1}\right),[i]\left(y_{1}\right)\right]\left(y_{2}, \ldots, y_{m}\right) \\
& =\left[[\alpha]\left([\alpha]\left(\ldots[\alpha]\left([\beta](f),[\beta]\left(x_{1}\right)\right), \ldots,[\beta]\left(x_{n}\right)\right), i\right)\right]\left(y_{1}, y_{2}, \ldots, y_{m}\right) .
\end{aligned}
$$

Now, by 2.3 , there exists $\left[s_{n}\right]_{n+1} \in F(I)$ such that, for all $f, x_{1}, \ldots, x_{n} \in V$,

$$
\left[s_{n}\right]\left(f, x_{1}, \ldots, x_{n}\right)=[\alpha]\left([\alpha]\left(\ldots[\alpha]\left([\beta]\left(x_{1}\right)\right), \ldots,[\beta]\left(x_{n}\right)\right), i\right) .
$$

But $\left[s_{n}\right]\left(f, x_{1}, \ldots, x_{n}\right) \neq *$ since $[\beta]_{1}$ and $[\alpha]_{2}$ are $*$-total, and, by the above, for any $y^{(m)} \in V^{m}, m>0$,

$$
\left[\left[s_{n}\right]\left(f, x_{1}, \ldots, x_{n}\right)\right]\left(y^{(m)}\right)=[f]\left(x_{1}, \ldots, x_{n}, y^{(m)}\right),
$$

just as desired.

THEOREM 2.7 ("FIXED POINT THEOREM"). Given any function $[g]_{n+1} \in F(I), n>0$ there exists $f \in V$ such that, for every $x^{(n)} \in V^{n},[f]\left(x^{(n)}\right)=[g]\left(f, x^{(n)}\right)$. 
Proof. Let $[g]_{n+1}$ be given. By 2.3 and 2.6, there exists $[w]_{n+1} \in F(I)$, such that, for all $y, x_{1}, \ldots, x_{n} \in V,[w]\left(y, x_{1}, \ldots, x_{n}\right)=[g]\left(\left[s_{1}\right](y, y), x_{1}, \ldots, x_{n}\right)$. But then, for all $x_{1}, \ldots, x_{n} \in V$,

$$
\begin{aligned}
{\left[\left[s_{1}\right](w, w)\right]\left(x_{1}, \ldots, x_{n}\right) } & =[w]\left(w, x_{1}, \ldots, x_{n}\right) & & \text { by } 2.6 \\
& =[g]\left(\left[s_{1}\right](w, w), x_{1}, \ldots, x_{n}\right) & & \text { by definition }[w] .
\end{aligned}
$$

Thus, it suffices to take $f=\left[s_{1}\right](w, w)$.

3. Theorems on gödelizations. The results on gödelizations presented in this section are, in general, not unexpected results; rather, they serve to establish that our formal definition of a gödelization is in line with our intuitive expectations. The first four results deal with properties of single gödelizations, the final three with relationships between different gödelizations.

We begin by proving that it is impossible to gödelize a set of total functions, or to give another interpretation, that we may not replace Axiom 1 by its negation.

THEOREM 3.1. In any U.R.S. $I=\langle U, G\rangle$ the set $F(I)$ contains 1-ary functions, other than $[*]_{1}$, which are not total; indeed, there exists $\theta \in V$ such that, for all $x \in V$, $[\theta](x)=*$.

Proof. We first show that there exists an element $q$ in $V$ such that $[q](q)=*$. By 2.3 there exists a function $[\delta]_{1} \in F(I)$ such that $[\delta](x)=[x](x)$ for all $x \in V$. But then, given $a, b \in V, a \neq b$, there exists, by 2.3 and 2.4 , a function $[q]_{1} \in F(I)$ such that, for all $x \in V$,

$$
\begin{aligned}
{[q](x) } & = \begin{cases}{\left[k_{b}\right](x)} & \text { if }[\delta](x) \neq b ;[\delta](x) \in V \\
{\left[k_{a}\right](x)} & \text { if }[\delta](x)=b\end{cases} \\
& = \begin{cases}b & \text { if }[x](x) \neq b, * \\
a & \text { if }[x](x)=b \\
* & \text { if }[x](x)=* .\end{cases}
\end{aligned}
$$

Now we must have $[q](q)=*$ for otherwise we get $[q](q)=a$ if and only if $[q](q)$ $=b \neq a$, a contradiction. But then it suffices to take $\theta=[\alpha]\left(k_{q}, k_{q}\right)$.

The next two results are elementary normal form theorems for U.R.S. A more sophisticated normal form theorem, analogous to Kleene's Normal Form Theorem, is presented in $\$ 5$.

THEOREM 3.2. Let $I=\langle U, G\rangle$ be a U.R.S. Then there exists a function $[\delta] \in F(1, I)$ such that for every function $[f] \in F(1, I)$ there exists a *-total function $[t] \in F(1, I)$ such that $[f]=[\delta] \circ[t]$; i.e., for all $x \in U,[f](x)=[\delta]([t](x))$.

Proof. Let $[f]_{1}$ be an arbitrary element of $F(1, I)$. Then, by 2.3 , there exists $f^{\prime} \in V$ such that for all $x, y \in V,\left[f^{\prime}\right](x, y)=[f](x)$. But then, by 2.6 , there exists $t=\left[s_{1}\right]\left(f^{\prime}\right) \in V$ such that, for all $x, y \in V,[t](x)=\left[s_{1}\right]\left(f^{\prime}, x\right) \neq *$, so $[t]_{1}$ is *-total, but $[t](x, y)=\left[s_{1}\right]\left(f^{\prime}, x, y\right)=\left[f^{\prime}\right](x, y)=[f](x)$. Thus, in particular, for every $x \in V$, 
$[[t](x)]([t](x))=[f](x)$, and so the desired result is achieved by taking $\delta$ such that $[\delta]_{1}$ is the function given by 2.3 , such that, for every $x \in V,[\delta](x)=[x](x)$.

The above function $[\delta]_{1}$, which is often called the diagonal function, can be employed in the theory of U.R.S. to build up a body of results analogous to those concerning creative sets in the theory of partial recursive functions (see [13]).

THEOREM 3.3. If $I=\langle U, G\rangle$ is a U.R.S., then there exists $g \in U$ such that, for all $x \in U$ and $y^{(n)} \in U^{n},[g]\left(x, y^{(n)}\right)=[x]\left(y^{(n)}\right)$.

Proof. Trivially, it suffices to take $g=i, i$ as in 2.1(b). Then, for all $x \in U$, and $y^{(n)} \in U^{(n)},[g]\left(x, y^{(n)}\right)=[[i](x)]\left(y^{(n)}\right)=[x]\left(y^{(n)}\right)$.

The next result is self-explanatory, but indicates that one-to-one enumerations of the partial recursive functions, such as Friedberg's [2], are not gödelizations in our sense. Note also that results analogous to a number of standard undecidability results, such as the halting problem and the equivalence problem, can be extracted from the proof given below.

THEOREM 3.4. In every U.R.S. $I=\langle U, G\rangle$ the map $G$ is infinite-to-one; i.e., for every $u \in U$, the set $W(u)=\{v \in U \mid[v]=[u]\}$ is an infinite set.

Proof. Say there exists an element $u_{0} \in U$ such that the set $W\left(u_{0}\right)$ is finite. Then, by $2.4(\mathrm{~b})$, we may find $0,1 \in V, 0 \neq 1$, and $\rho \in U$ such that, for every $x \in V$,

$$
\begin{aligned}
{[\rho](x) } & =0 & & \text { if } x \in W \\
& =1 & & \text { if } x \notin W .
\end{aligned}
$$

Now we may employ $[\rho]$ to tell, for any $f, y \in V$, whether or not $[f](y)=\left[u_{0}\right](y)$. For, by 2.4 , there exists $\tau \in U$ such that, for all $f, y$, and $x$ in $V$,

But then, for any $f, y \in V$,

$$
\begin{aligned}
& {[[\tau](f, y)](x)=\left[u_{0}\right](x) \text { if } x \neq y} \\
& =[f](x) \text { if } x=y \text {. }
\end{aligned}
$$

$$
[f](y)=\left[u_{0}\right](y) \Leftrightarrow[\tau](f, y) \in W\left(u_{0}\right) \Leftrightarrow[\rho]([\tau](f, y))=0 .
$$

Now we may employ $[\rho]$ and $[\tau]$ together to determine, for arbitrary $g \in V$, whether or not $[g](g)=*$. For, if we pick some element $v_{0} \in V$, then either $\left[u_{0}\right]\left(v_{0}\right)$ $=*$ or $\left[u_{0}\right]\left(v_{0}\right)=w \in V$. In the first case, let $r \in V$, by 2.3, such that, for all $g, x \in V$, $[[r](g)](x)=[g](g)$ and, in the second case, let $r \in V$ such that, for all $g, x \in V$, $[[r](g)](x)=\left[k_{w}\right]([g](g))$. Then, in the first case, for any $g \in V$,

$$
\begin{array}{rlrl}
{[\rho]\left([\tau]\left([r](g), v_{0}\right)\right)} & =0 & & \text { if }[g](g)=* \\
& =1 & \text { if }[g](g) \neq *,
\end{array}
$$

and, in the second case,

$$
\begin{array}{rlr}
{[\rho]\left([\tau]\left([r](g), v_{0}\right)\right)} & =0 & \text { if }[g](g) \neq * \\
& =1 & \text { if }[g](g)=* .
\end{array}
$$


In either case, it follows from 2.3 and the arbitrariness of the choice of 0 and 1 , that there exists $\mu \in V$ such that, for any $g \in V$

$$
\begin{aligned}
{[\mu](g) } & =0 & & \text { if }[g](g)=* \\
& =1 & & \text { if }[g](g) \neq * .
\end{aligned}
$$

But then, by 2.3 and 3.1 there exists $\mu * \in V$ such that, for all $g \in V$,

$$
\begin{aligned}
& {[\mu *](g)=\left[[\psi]\left(0,0, k_{\theta},[\mu](g)\right)\right](g)=0 \text { if }[g](g)=*} \\
& =* \text { if }[g](g) \neq * \text {. }
\end{aligned}
$$

This, however, leads to a contradiction since we get $[\mu *](\mu *)=* \Leftrightarrow[\mu *](\mu *) \neq *$. Thus, for every $u \in U$, we must have $W(u)$ infinite.

We turn now to theorems dealing with different gödelizations of the same set of functions. We first show that $F(1, I)$ completely determines $F(I)$ irrespective of the choice of $G$. It follows, in particular, from this result that no matter how we gödelize the 1-ary partial recursive functions (in keeping with our axioms), the set of $n$-ary functions we get by iteration is just the set of $n$-ary partial recursive functions.

THeOREM 3.5. Let $I=\langle U, G\rangle$ and $I^{\prime}=\left\langle U, G^{\prime}\right\rangle$ be two U.R.S. with domain $U$. Then $G(U)=G^{\prime}(U)$, i.e., they have the same 1-ary functions, if and only if $F(I)=F\left(I^{\prime}\right)$.

Proof. That $F(I)=F\left(I^{\prime}\right)$ implies $G(U)=G^{\prime}(U)$ is immediate.

Now say $G(U)=G^{\prime}(U)$. Then, to prove $F(I)=F\left(I^{\prime}\right)$ we claim it suffices to show that $F(I)$ and $F\left(I^{\prime}\right)$ contain the same 2-ary functions. To see this, let us, for each $u \in U$, write $[u]$ for $G(u)$ and $[u]^{\prime}$ for $G^{\prime}(u)$. From the assumption that every 2-ary function in $F(I)$ is in $F\left(I^{\prime}\right)$, it follows, from 3.3, that there exists $[g]_{2}^{\prime} \in F\left(I^{\prime}\right)$ such that, for all $x, y \in V,[g]^{\prime}(x, y)=[i](x, y)=[x](y)$. But then, for any $u \in U$, integer $n>0$, and $x^{(n)}=\left\langle x_{1}, \ldots, x_{n}\right\rangle \in U^{n}$,

$$
\begin{aligned}
{[u]_{n}\left(x_{1}, \ldots, x_{n}\right) } & =\left[\ldots\left[[u]\left(x_{1}\right)\right]\left(x_{2}\right) \ldots\right]\left(x_{n}\right) \\
& =[g]^{\prime}\left(\ldots[g]^{\prime}\left([g]^{\prime}\left(u, x_{1}\right), x_{2}\right) \ldots, x_{n}\right) \\
& =[h]_{n}^{\prime}\left(x_{1}, \ldots, x_{n}\right)
\end{aligned}
$$

for some $h \in U$, by 2.3 (as stated for $I^{\prime}$ ). Hence, $[u]_{n}=[h]_{n}^{\prime} \in F\left(I^{\prime}\right)$, and so $F(I)$ $\subset F\left(I^{\prime}\right)$. By symmetry $F\left(I^{\prime}\right) \subset F(I)$ and so $F\left(I^{\prime}\right)=F(I)$.

It remains to show then that $F(I)$ and $F\left(I^{\prime}\right)$ contain the same 2-ary functions when $G(U)=G^{\prime}(U)$. We shall achieve this end by, in effect, reducing the 2-ary functions in $F\left(I^{\prime}\right)$ to 1 -ary functions by means of special pairing functions.

Let $c, d$ be distinct fixed elements of $V$. Then where $\lambda=[\alpha]\left(i, k_{c}\right)$ and $\lambda_{2}=[\alpha]\left(i, k_{d}\right)$ we have, for all $x, y \in V$,

$$
\left[\lambda_{1}\right]([\psi](c, x, y))=y, \quad\left[\lambda_{2}\right]([\psi](c, x, y))=x .
$$


Now, since $G(U)=G^{\prime}(U)$, there exist functions $\left[\lambda_{1}^{\prime}\right]^{\prime}$ and $\left[\lambda_{2}^{\prime}\right]^{\prime}$ in $F\left(I^{\prime}\right)$ such that $\left[\lambda_{1}^{\prime}\right]^{\prime}=\left[\lambda_{1}\right]$ and $\left[\lambda_{2}^{\prime}\right]^{\prime}=\left[\lambda_{2}\right]$.

Let $\left[r^{\prime}\right]_{2}^{\prime} \in F\left(I^{\prime}\right)$, to see that $\left[r^{\prime}\right]_{2}^{\prime}$ is also in $F(I)$, consider that, by 2.3 (as applied to $\left.I^{\prime}\right)$, there must exist $\left[w^{\prime}\right]_{1}^{\prime} \in F\left(I^{\prime}\right)$ such that, for every $z \in V$,

$$
\left[w^{\prime}\right]^{\prime}(z)=\left[r^{\prime}\right]^{\prime}\left(\left[\lambda_{1}\right]^{\prime}(z),\left[\lambda_{2}\right]^{\prime}(z)\right) \text {. }
$$

But then, since $G(U)=G^{\prime}(U)$, there must exist $[w]_{1} \in F(I)$ such that $[w]_{1}=\left[w^{\prime}\right]_{1}^{\prime}$. And so, by 2.3 , there exists $[r]_{2} \in F(I)$ such that, for every $x, y \in U$,

$$
\begin{aligned}
{[r](x, y) } & =[w]([\psi](c, x, y)) \text { which by the above } \\
& =\left[w^{\prime}\right]^{\prime}([\psi](c, x, y)) \\
& =\left[r^{\prime}\right]^{\prime}\left(\left[\lambda_{1}^{\prime}\right]^{\prime}([\psi](c, x, y)),\left[\lambda_{2}^{\prime}\right]^{\prime}([\psi](c, x, y))\right) \\
& =\left[r^{\prime}\right]^{\prime}\left(\left[\lambda_{1}\right]([\psi](c, x, y)),\left[\lambda_{2}\right]([\psi](c, x, y))\right) \\
& =\left[r^{\prime}\right]^{\prime}(x, y) .
\end{aligned}
$$

Hence, $\left[r^{\prime}\right]_{2}^{\prime}=[r]_{2} \in F(I)$, so every 2-ary function in $F\left(I^{\prime}\right)$ is in $F(I)$ and, by symmetry, $F(I)$ and $F\left(I^{\prime}\right)$ contain the same 2-ary functions and we are done.

REMARK. As we will show subsequently, the stronger statement, "if $F(1, I)$ and $F\left(1, I^{\prime}\right)$ contain the same $*$-total functions, then $F(I)=F\left(I^{\prime}\right)$ ", is false.

The following result corresponds to the intuitive idea that given any two gödelizations of the same set of functions, there must exist an effective way to get from one gödelization to the other. Applying this result to the partial recursive functions, we see that the class of gödelizations for the partial recursive functions which we have defined is precisely Rogers' class of "fully effective numberings" [4].

THEOREM 3.6. If $I=\langle U, G\rangle$ and $I^{\prime}=\left\langle U, G^{\prime}\right\rangle$ are two U.R.S. with domain $U$ and $F(I)=F\left(I^{\prime}\right)$, then there exists $[\rho]_{1} \in F(I),[\rho]_{1} *$-total, such that $G \circ[\rho]_{1}=G^{\prime}$.

Proof. As in 3.5, for each $u \in U$, let $[u]$ denote $G(u)$ and $[u]^{\prime}$ denote $G^{\prime}(u)$.

Let $\left[i^{\prime}\right]_{1}^{\prime}$ be the identity function in $F\left(I^{\prime}\right)$ as given by $2.1(\mathrm{~b})$. Then, of course, for all $u, x \in U,\left[i^{\prime}\right]^{\prime}(u, x)=\left[\left[i^{\prime}\right]^{\prime}(u)\right]^{\prime}(x)=[u]^{\prime}(x)$. But, since $F(I)=F\left(I^{\prime}\right)$, there must exist $\mu \in U$ such that $[\mu]_{2}=\left[i^{\prime}\right]_{2}^{\prime}$, and clearly, for all $u, x \in U$,

$$
\left(\left(G \circ[\mu]_{1}\right)(u)\right)(x)=[[\mu](u)](x)=\left[i^{\prime}\right]^{\prime}(u, x)=[u]^{\prime}(x)=\left(G^{\prime}(u)\right)(x) .
$$

If now $[\mu]_{1}$ is total, we may take $\rho=\mu$, and, if $[\mu]_{1}$ is not total, then we may take $\rho$, by 2.3 , such that, for any $u \in V,[\rho](u)=[\alpha]\left([\alpha]\left(k_{\mu},[\beta](u)\right), i\right)$, which is not equal $*$ since $[\alpha]_{2}$ and $[\beta]_{1}$ are both $*$-total; but then, for any $u, x \in U$ we have

$$
\begin{aligned}
{[\rho](u, x) } & =\left[[\rho]\left([\rho]\left(k_{\mu},[\beta](u)\right), i\right)\right](x) \\
& =\left[\left[[\rho]\left(k_{\mu},[\beta](u)\right)\right](x)\right]([i](x)) \\
& =\left[\left[\left[k_{\mu}\right](x)\right]([\beta](u, x))\right]([i](x)) \\
& =[\mu](u, x) .
\end{aligned}
$$


The following result serves as a converse to the preceding one and provides means for producing new gödelizations from existing ones. It follows easily from this result that, given a U.R.S. $I=\langle U, G\rangle$, then $F(I)$ has Card $(U)$ gödelizations.

THEOREM 3.7. Let $I=\langle U, G\rangle$ be a U.R.S. If $[f]_{1}$ and $[g]_{1}$ are total functions in $F(I)$ such that, for every $u$, and $x$ in $V,[f]([g](u), x)=[u](x)$, then the structure $I^{\prime}=\left\langle U, G^{\prime}\right\rangle$, where $G^{\prime}=G \circ[f]_{1}$ is a U.R.S. and $F\left(I^{\prime}\right)=F(I)$, where $F\left(I^{\prime}\right)$ is the set of functions generated from $I^{\prime}$ by iteration.

Proof. Given $u \in U$, let $[u]$ denote $G(u)$ and let $[u]^{\prime}$ denote $G^{\prime}(u)$.

We first prove that $F\left(I^{\prime}\right) \subseteq F(I)$. To see this, let $[u]_{n}^{\prime} \in F\left(I^{\prime}\right)$. Then, by definition, for every $x^{(n)}=\left\langle x_{1}, x_{2}, \ldots, x_{n}\right\rangle \in U^{n}$,

$$
[u]_{n}^{\prime}\left(x^{(n)}\right)=\left[[f]\left(\ldots\left[[f]\left([[f](u)]\left(x_{1}\right)\right)\right]\left(x_{2}\right) \ldots\right)\right]\left(x_{n}\right),
$$

from which it follows immediately, by 2.3 , that there exists $v \in V$ such that $[v]_{n}=$ $[u]_{n}^{\prime}$. Thus, $F\left(I^{\prime}\right) \subseteq F(I)$.

To see now that $[u]_{n} \in F(I)$ implies $[u]_{n} \in F\left(I^{\prime}\right)$, we proceed by induction on $n$. For $n=1$ we have that if $[u]_{1} \in F(I)$, then, by the conditions of the theorem, $[[g](u)]_{1}^{\prime}=[u]_{1}$. Now, assuming the result is proved up to $n=k$, let $[u]_{k+1} \in F(I)$. Then, for any $x^{(k+1)}=\left\langle x_{1}, \ldots, x_{k}, x_{k+1}\right\rangle \in U^{k+1}$, we have

$$
\begin{aligned}
{[u]_{k+1}\left(x^{(k+1)}\right) } & =\left[[u]\left(x_{1}, \ldots, x_{k}\right)\right]\left(x_{k+1}\right) \\
& =[f]\left([g]\left([u]\left(x_{1}, \ldots, x_{k}\right)\right), x_{k+1}\right)
\end{aligned}
$$

by the choice of $[f]$ and $[g]$. Then, by the definition of $G^{\prime},(1)$ equals

$$
\left[[g]\left([u]\left(x_{1}, \ldots, x_{k}\right)\right)\right]^{\prime}\left(x_{k+1}\right) .
$$

But, by 2.3, there exists $v \in U$ such that, for all $x^{(k)} \in U^{k},[v]\left(x^{(k)}\right)=[g]\left([u]\left(x^{(k)}\right)\right)$, and, by the induction hypothesis, there exists $v^{\prime} \in U$ such that $\left[v^{\prime}\right]_{k}^{\prime}=[v]_{k}$. Hence, (2) equals

$$
\left[\left[v^{\prime}\right]\left(x_{1}^{\prime}, \ldots, x_{k}\right)\right]^{\prime}\left(x_{k+1}\right)=\left[v^{\prime}\right]^{\prime}\left(x_{1}, \ldots, x_{k+1}\right)
$$

by the definition of $(k+1)$-ary function. Thus, $F(I) \subseteq F\left(I^{\prime}\right)$ and so $F\left(I^{\prime}\right)=F(I)$.

It remains then to show that $I^{\prime}=\left\langle U, G^{\prime}\right\rangle$ is a U.R.S. That it satisfies Axiom 1 is trivial. That it satisfies Axiom 3 follows from $F\left(I^{\prime}\right)=F(I)$. Thus, it remains to prove Axiom 2. What we must show is that there exists $\alpha \in V$ such that, for any $p, q$, and $x$ in $V,\left[\alpha^{\prime}\right](p, q) \neq *$ and $\left[\alpha^{\prime}\right](p, q, x)=\left[[p]^{\prime}(x)\right]^{\prime}\left([q]^{\prime}(x)\right)$. That is,

$$
\left[\alpha^{\prime}\right]^{\prime}(p, q, x)=[[f]([[f](p)](x))]([[f](q)](x)) .
$$

To see this, let $[t]_{2}$ be the element of $F(I)$, whose existence is established by 2.3, such that, for all $p$ and $q$ in $V,[t](p, q)=[g]([\alpha]([\gamma](f,[f](p)),[f](q))$. From $F\left(I^{\prime}\right)=F(I)$, it follows that there exists $\left[\alpha^{\prime}\right]_{2}^{\prime} \in F\left(I^{\prime}\right)$ such that $\left[\alpha^{\prime}\right]_{2}^{\prime}=[t]_{2}$. Furthermore, 
from the fact that $[g]_{1},[\alpha]_{2},[\gamma]_{2}$ and $[f]_{1}$ are all total, it follows that $\left[\alpha^{\prime}\right]_{2}^{\prime}$ is total. But then, for all $p, q$ and $x$ in $V$,

$$
\begin{aligned}
{\left[\alpha^{\prime}\right]^{\prime}(p, q, x) } & =\left[\left[\alpha^{\prime}\right]^{\prime}(p, q)\right]^{\prime}(x) \\
& =[[t](p, q) \\
& =[f]([t](p, q), x) \\
& =[f]([g]([\alpha]([\gamma](f,[f](p)),[f](q)), x) \\
& =[[\alpha]([\gamma](f,[f](p)),[f](q))](x) \\
& =[[f]([[f](p)](x))]([[f](q)](x)) \\
& =\left[[p]^{\prime}(x)\right]^{\prime}\left([q]^{\prime}(x)\right)
\end{aligned}
$$

as desired.

4. Sets, predicates, recursion and minimality. In this section, we present a number of definitions and results which will be needed in the coming sections and which are also of interest in that they generalize to U.R.S. more of the conventional theory of computability.

We shall wish to speak of sets and predicates which are representable within U.R.S. To this end, we directly generalize the notions of computable and semicomputable sets from recursive function theory.

Let $I=\langle U, G\rangle$ be a U.R.S.; let 0 , and 1 be two distinct elements of $V$; let $m$ be a nonnegative integer and let $A \subseteq V^{m}$. Then we say that $A$ is computable (resp. semicomputable) if the characteristic function of $A$,

$$
\begin{aligned}
C_{A}\left(x^{(m)}\right) & =0 & & \text { if } x^{(m)} \in A \\
& =1 & & \text { if } x^{(m)} \notin A
\end{aligned}
$$

(resp. the semicharacteristic function of $A$

$$
\begin{aligned}
& S_{A}\left(x^{(m)}\right)=0 \quad \text { if } x^{(m)} \in A \\
& \left.=* \quad \text { if } x^{(m)} \notin A\right)
\end{aligned}
$$

is in $F(I)$.

We say that an $n$-ary predicate $P$ on $V$ is computable (resp. semicomputable) if the set $\left\{x^{(n)} \mid P\left(x^{(n)}\right)\right\}$ is computable (resp. semicomputable).

Note. We use the notion of semicomputable set (from Davis [1]) rather than the more common notion of recursively enumerable set since these two notions, while equivalent in the theory of partial recursive functions, are not equivalent in arbitrary U.R.S. (see Theorem 6.13), and in these cases, semicomputability appears to be the more intuitively natural notion.

From the following theorem, it is easily seen that the computable sets are closed under intersection, union, and complementation, and that the semicomputable sets are closed under intersection. This, of course, implies corresponding results for the 
conjunction, alternation and negation of computable and semicomputable predicates. Note that the tables for $\wedge$ and $\vee$ are asymmetric. Thus, the evaluation of an expression involving semicomputable predicates may be 0 or 1 even though some of the predicates take the value $*$. This asymmetry will be of use in $\$ 5$.

THEOREM 4.1. Let $I=\langle U, G\rangle$ be a U.R.S. and let $[p]_{n},[q]_{n} \in F(n, I)$ such that for every $x^{(n)} \in V^{n},[p]\left(x^{(n)}\right),[q]\left(x^{(n)}\right) \in\{0,1, *\}$. Then there exist $\left[f_{p \wedge q}\right]_{n},\left[f_{p \vee q}\right]_{n}$, and $\left[f_{\sim p}\right]_{n}$ in $F(I)$ in accordance with the following tables:

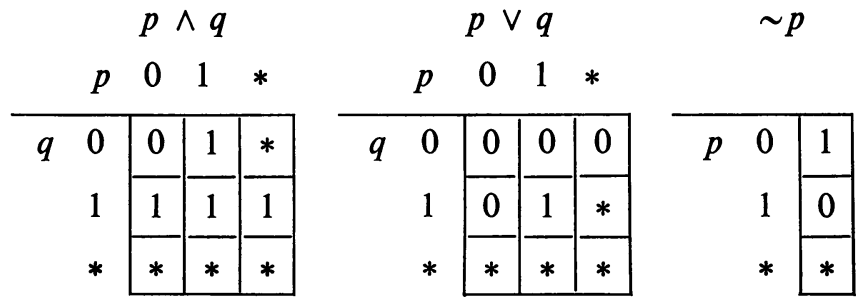

Proof. The desired result follows from 2.5 since the functions can be expressed by cases as follows:

$$
\begin{aligned}
{\left[f_{p \wedge q}\right]\left(x^{(n)}\right) } & =[q]\left(x^{(n)}\right) & & \text { if }[p]\left(x^{(n)}\right)=0 \\
& =1 & & \text { if }[p]\left(x^{(n)}\right)=1 \\
& =* & & \text { if }[p]\left(x^{(n)}\right)=* \\
{\left[f_{p \vee q}\right]\left(x^{(n)}\right) } & =[q]\left(x^{(n)}\right) & & \text { if }[p]\left(x^{(n)}\right)=1 \\
& =0 & & \text { if }[p]\left(x^{(n)}\right)=0 \\
& =* & & \text { if }[p]\left(x^{(n)}\right)=* \\
{\left[f_{\sim p}\right]\left(x^{(n)}\right) } & =0=\left[k_{0}\right]\left(\left[u_{n, 1}\right]\left(x^{(n)}\right)\right. & & \text { if }[p]\left(x^{(n)}\right)=1 \\
& =1=\left[k_{1}\right]\left(\left[u_{n, 1}\right]\left(x^{(n)}\right)\right. & & \text { if }[p]\left(x^{(n)}\right)=0 \\
& =* & & \text { if }[p]\left(x^{(n)}\right)=* .
\end{aligned}
$$

The following simple results on computable and semicomputable sets in U.R.S. are worth noting.

Proposition 4.2. 1. A set $A \subset V$ is semicomputable if and only if there exists $[f] \in F(I)$ such that $A=\{x \mid[f](x) \neq *\}$.

2. There exists an enumeration with repetitions of the set of semicomputable sets i.e., a map of $V$ onto the set of semicomputable sets (we shall generally write $\omega_{u}$ for the semicharacteristic function $\omega(u))$.

3. If $A \subset V$ is a computable set, then both $A$ and $V-A$ are semicomputable sets.

4. All finite and cofinite sets $A \subset V$ are computable. Note: It has been shown by Strong [8] that there exist U.R.S. in which these sets are the only computable subsets of $V$.

5. The equality predicate is computable. 
Proof. 1. Given $[f]_{1}$, let $\left[k_{0}\right]$ be as in 2.1. Then $A$ has the semicharacteristic function $\left[k_{0}\right] \circ[f]=\left[[\gamma]\left(k_{0}, f\right)\right]$.

2. For every $u \in V$, let $\omega_{u}=\left[[\gamma]\left(k_{0}, u\right)\right]$.

3. Follows from 2.4 and 3.1 .

4. Follows from 2.4 .

5. Take $[e](x, y)=[\psi](x, 1,0, y)$.

We turn now to the extending of the operations of primitive recursion and minimalization to arbitrary U.R.S. What we do is show that, in any U.R.S. $I=\langle U, G\rangle$, we can pick subsets of $V$ which are analogous to the integers and with respect to which we can perform the operations of primitive recursion and minimalization. These sets, which need not be either computable or semicomputable with respect to $I$, are defined as follows:

Given $[f] \in F(1, I)$, let $[f]^{0}=[i],[f]^{1}=[f]$ and, for $n \geqq 1$, let $[f]^{n+1}=[f] \circ[f]^{n}$. That $[f] \in F(1, I)$ implies $[f]^{n} \in F(1, I)$ follows from 2.3.

Let $I=\langle U, G\rangle$ be a U.R.S. We say that a pair $\langle[s], z\rangle([s] \in F(1, I), z \in V)$ is a successor pair for $I$ if for all integers $n, m \geqq 0,[s]^{n}(z)=[s]^{m}(z) \Leftrightarrow n=m$. If $\langle[s], z\rangle$ is a successor pair for $I$, then the set $N=\left\{[s]^{n}(z) \mid n\right.$ is an integer, $\left.n \geqq 0\right\}$ is called the successor set of $\langle[s], z\rangle,[s]$ is called the successor function and $z$ is called the zero.

As an example of a successor pair, we have, for the U.R.S. of the partial recursive functions, the pair $\langle s, 0\rangle$ where $s$ is the "standard successor function", $s(n)=n+1$. For an arbitrary U.R.S., it is easy to show that the pair $\langle[\beta], \beta\rangle(\beta$ as in 2.1 ) is a successor pair. In applying the results of this section, we shall be most concerned with cases in which the successor set $N$ is a computable or semicomputable set (with respect to the given U.R.S.); however, in this section, only our final result employs a semicomputable successor set. The question of whether or not there exist U.R.S. which do not contain any computable successor sets was left open in [10]. This question has since been answered in the affirmative by Strong [8]. More recently Friedman [3] has shown that there exist U.R.S. which do not even contain semicomputable successor sets.

We first show that given a successor pair for a U.R.S. $I$ that $F(I)$ is closed with respect to primitive recursion.

THEOREM 4.3. Let $I=\langle U, G\rangle$ be a U.R.S. and let $\langle[s], z\rangle$ be a successor pair for $I$ with successor set $N$. Then, for every pair of functions $[f]_{k+2}$ and $[g]_{k}$ in $F(I)$, there exists a function $[r]_{k+1}$ in $F(I)$ such that, for all $x^{(k)} \in V^{k},[r]\left(z, x^{(k)}\right)=[g]\left(x^{(k)}\right)$ and, for all integers $n \geqq 0$,

$$
[r]\left([s]^{n+1}(z), x^{(k)}\right)=[f]\left(x^{(k)},[s]^{n}(z),[r]\left([s]^{n}(z), x^{(k)}\right)\right) .
$$

Proof. For the sake of simplicity, take $k=1$. Now say we can find a function $[\lambda]_{5} \in F(I)$ such that, for all $w_{1}, \ldots, w_{5} \in V$,

$$
\begin{aligned}
{[\lambda]\left(w_{1}, \ldots, w_{5}\right) } & =w_{3} & & \text { if } w_{2}=w_{4} \\
& =[\lambda]\left(w_{1},[s]\left(w_{2}\right),\left[w_{1}\right]\left(w_{5}, w_{2}, w_{3}\right), w_{4}, w_{5}\right) & & \text { if } w_{2} \neq w_{4} .
\end{aligned}
$$


Then, taking $[r]_{2}$ to be such that $[r]\left([s]^{n}(z), x\right)=[\lambda] f,\left(z,[g](x),[s]^{n}(x), x\right)$, it is easily seen by induction on $n$, that we get the desired result.

The existence of the desired function $[\lambda]_{5}$ may be established as follows:

Let $0,1 \in V, 0 \neq 1$, and let [ $t]$ be the function, given by 2.5 , such that, for any $w_{0}, w_{1}, \ldots, w_{5} \in V,[t]\left(w_{0}, w_{1}, \ldots, w_{5}\right)=\left[\left[h_{2},{ }_{6}\right]\left(t_{0}, t_{1}, t_{2}\right)\right]\left(w_{0}, w_{1}, \ldots, w_{5}\right)$ where

$$
\begin{aligned}
{\left[t_{0}\right]\left(w_{0}, w_{1}, \ldots, w_{5}\right)=[\psi]\left(w_{2}, 1,0, w_{4}\right)=0 } & \text { if } w_{2}=w_{4} \\
=1 & \text { if } w_{2} \neq w_{4}
\end{aligned}
$$

by 2.3 and Axiom $1 .\left[t_{1}\right]\left(w_{0}, w_{1}, \ldots, w_{5}\right)=w_{3}$ by 2.1 . Lastly,

$$
\left[t_{2}\right]\left(w_{0}, w_{1}, \ldots, w_{5}\right)=\left[w_{0}\right]\left(w_{1},[s]\left(w_{2}\right),\left[w_{1}\right]\left(w_{5}, w_{2}, w_{3}\right), w_{4}, w_{5}\right)
$$

by 2.3. But then, by 2.7 , there exists $\lambda \in V$ such that for all $\left\langle w_{1}, \ldots, w_{5}\right\rangle \in V$, $[\lambda]\left(w_{1}, \ldots, w_{5}\right)=[t]\left(\lambda, w_{1}, \ldots, w_{5}\right)$, and from the definition of $[t]_{6}$ (i.e., from 2.5), we see that $\left[\lambda_{5}\right]$ is precisely the desired function.

In our most important use of the above result in this paper 5.3, we shall employ it to define two functions simultaneously in terms of each other. The validity of such "joint-recursive-definitions" follows readily from the above result if we employ the simple device of combining the two functions into a single function; e.g., to define say 1-ary functions $f:$ and $g: N \rightarrow V$ where $f\left([s]^{n+1}(z)\right)$ depends on $g\left([s]^{n}(z)\right)$ and vice versa, it suffices to define a single 2-ary function $h$ such that, for all $n \in N$

$$
\begin{aligned}
h(x, n)=f(n) & \text { if } x=0 \\
=g(n) & \text { if } x \neq 0
\end{aligned}
$$

We shall now prove two theorems on minimalization. The first is a general result, good for all successor pairs. The second requires that the successor set $N$ be at least semicomputable. While minimalization is traditionally defined with respect to total functions, we here adopt a trivial generalization which will be more in accord with our applications.

Let $I=\langle U, G\rangle$ be a U.R.S. with successor pair $\langle[s], z\rangle$ and let $c \in V$. Then, a function $[f]_{n+1},(n \geqq 1)$, will be said to be $c$-consistent if, for every $x^{(n)} \in V^{n}$, if there exists a least integer $m$ such that $[f]\left([s]^{m}(z), x^{(n)}\right)=c$, then for all $m^{\prime}, 0 \leqq m^{\prime} \leqq m$, $[f]\left([s]^{m^{\prime}}(z), x^{(n)}\right) \neq *$.

THEOREM 4.4. Let $I=\langle U, G\rangle$ be a U.R.S. with successor pair $\langle[s], z\rangle$ and let $c$ be a fixed element of $V$. Then for each integer $n>0$ there exists a function $\left[\mu_{n}\right]_{n+1}$ in $F(I)$ such that, for every c-consistent function $[f]_{n+1} \in F(I)$, and every $x^{(n)} \in V^{n}$, $\left[\mu_{n}\right]\left(f, x^{(n)}\right)=$ the least element $[s]^{m}(z)$ of $N$ such that $[f]\left([s]^{m}(z), x^{(n)}\right)=c$, if such exists. (Note that no value is specified where no such $[s]^{m}(z)$ exists.) 
Proof. For simplicity, take $n=1$. We claim it will suffice to find a function $[\lambda]_{3}$ in $F(I)$ such that, for all $w_{1}, w_{2}, w_{3} \in V$,

$$
\begin{aligned}
{[\lambda]\left(w_{1}, w_{2}, w_{3}\right) } & =w_{1} & & \text { if }\left[w_{2}\right]\left(w_{1}, w_{3}\right)=c, \\
& =[\lambda]\left([s]\left(w_{1}\right), w_{2}, w_{3}\right) & & \text { if }\left[w_{2}\right]\left(w_{1}, w_{3}\right) \neq c .
\end{aligned}
$$

For, if such $[\lambda]_{3}$ exists, then taking $\left[\mu_{1}\right]$ such that, for all $f$ and $x$ in $V,\left[\mu_{1}\right](f, x)$ $=[\lambda](z, f, x)$, we see by inspection that we get the desired result. (In effect, $[\lambda]_{3}(z, f, x)$ successively computes $[f]\left([s]^{n}(z), x\right)$ for $n=0,1,2, \ldots$ until the desired element of $N$, if such exists, is reached.)

The existence of $[\lambda]_{3}$ may be established as follows:

Let $0,1 \in V, 0 \neq 1$, and take $[t]_{4}$, by 2.5 , such that, for all $w_{0}, w_{1}, w_{2}, w_{3} \in V$,

$$
[t]\left(w_{0}, \ldots, w_{3}\right)=\left[\left[h_{2,4}\right]\left(t_{0}, t_{1}, t_{2}\right)\right]\left(w_{0}, \ldots, w_{3}\right)
$$

where

$$
\begin{aligned}
& {\left[t_{0}\right]\left(w_{0}, \ldots, w_{3}\right)=[\psi]\left(z, 1,0,\left[w_{2}\right]\left(w_{1}, w_{3}\right)\right)=0 \text { if }\left[w_{2}\right]\left(w_{1}, w_{3}\right)=c} \\
& =1 \text { if }\left[w_{2}\right]\left(w_{1}, w_{3}\right) \neq c ; \\
& {\left[t_{1}\right]\left(w_{0}, \ldots, w_{3}\right)=w_{1}}
\end{aligned}
$$

and

$$
\left[t_{2}\right]\left(w_{0}, \ldots, w_{3}\right)=\left[w_{0}\right]\left([s]\left(w_{1}\right), w_{2}, w_{3}\right) .
$$

But then; by the fixed point Theorem 2.7, there exists $\lambda \in V$ such that, for all $w_{1}, w_{2}, w_{3} \in V,[\lambda]\left(w_{1}, w_{2}, w_{3}\right)=[t]\left(\lambda, w_{1}, w_{2}, w_{3}\right)$ which is easily seen, by inspection, to be the desired function.

Inspection of the above theorem will show that we have not specified the value of $[\mu](f, x)$ when there is no $[s]^{k}(z) \in N$ such that $[f]\left([s]^{k}(z), x\right)=c$. In order to mimic conventional minimization, we would want $[\mu](f, x)$ to have the value $*$ under these circumstances. However, it would appear that the fixed point theorem (as used to develop $\lambda$ ) is not strong enough to guarantee this result. On the other hand, if $N$ is a semicomputable successor set, then we are able to get the desired result.

THEOREM 4.5. Let $I=\langle U, G\rangle$ be a U.R.S. with successor pair $\langle[s], z\rangle$ with semicomputable successor set $N$ and let $c$ be a fixed element of $V$. Then for each integer $n>0$, there exists a function $\left[\mu_{n} *\right]_{n+1}$ in $F(I)$ such that, for every c-consistent function $[f]_{n+1} \in F(I)$, and every $x^{(n)} \in V^{n}$,

$$
\begin{aligned}
{\left[\mu_{n} *\right]\left(f, x^{(n)}\right) } & =\text { the least element }[s]^{k}(z) \in N \text { such that }[f]\left([s]^{k}(z), x^{(n)}\right)=c, \\
& \text { if such exists; } \\
& =* \text { otherwise. }
\end{aligned}
$$

Proof. For simplicity, we again take $n=1$. Let $\left[\mu_{1}\right]$ be as in the preceding theorem. What we wish to do is make $\left[\mu_{1} *\right]$ such that $\left[\mu_{1} *\right](f, x)=\left[\mu_{1}\right](f, x)$ except when 
$\left[\mu_{1}\right](f, x) \neq *$ but $[f]\left(\left[\mu_{1}\right](f, x), x\right) \neq c$ or $\left[\mu_{1}\right](f, x) \notin N$, in which case we want $\left[\mu_{1} *\right](f, x)=*$. This may clearly be accomplished by use of 2.5 .

Rather than use the above notation, $\left[\mu_{n} *\right]\left(f, x^{(n)}\right)$, in the remainder of the paper, we shall employ the more suggestive and complete notation, $\min _{y \in N}[f]\left(y, x^{(n)}\right)=c$.

5. The construction of U.R.S. and highly constructible U.R.S. We begin this section by presenting a means for "constructing" U.R.S. on arbitrary infinite domains. Using this "construction", we prove that the functions recursive in $f$ form the function set of a U.R.S. This proof, and the new gödelization which it presents, allows us to show that the results given in the remainder of the paper apply to the partial recursive functions. We next present a fundamental lemma which presents sufficient conditions under which the "construction" given in the first theorem can be carried out within a U.R.S. On the basis of these results, we develop and investigate a special class of U.R.S., the highly constructible U.R.S. This class includes the U.R.S. of partial recursive functions. The highly constructible U.R.S. enjoy a number of properties important in the theory of computability which are not enjoyed by arbitrary U.R.S. In addition, the members of this class will form the basis of the intrinsic theory of relative computability developed in $\$ 6$.

A function $f: U^{n} \rightarrow U$ is said to be $*$-admissible if and only if, for all $x^{(n)} \in U^{n}-V^{n}$, we have $f\left(x^{(n)}\right)=*$.

THEOREM 5.1. Given an infinite set $U$, a designated element $*$ of $U$, and a set $F$ of 1-ary *-admissible functions of $U$ into $U$ where $\operatorname{Card}(F) \leqq C a r d(U)$, then there exists a U.R.S. $I=\langle U, G\rangle$ such that $F \subseteq F(I)$.

Proof. Let $V=U-\{*\}$. Let $\alpha$ and $\psi$ be two arbitrary elements of $V$. Let $V_{0}, V_{1}, \ldots, V_{6}$ be seven disjoint subsets of $V$ such that, $V_{0}=\{\alpha, \psi\}$, Card $\left(V_{1}\right)$ $\geqq \operatorname{Card}(F)$, and, for $2 \leqq i \leqq 6$, we have Card $\left(V_{i}\right)=\operatorname{Card}(V)$. Let $V_{7}=V-\bigcup_{i=0}^{6} V_{i}$. Using these sets, we will now form functions from which we can develop a gödelization $G: U \rightarrow U^{U}$ which will satisfy the axioms. In particular, we will have $G(\alpha)=[\alpha], G(\psi)=[\psi] ; V_{1}$ will contain gödel numbers for all of the elements of $\boldsymbol{F}$; and $V_{2}$ through $V_{6}$ will contain gödel numbers corresponding to functions of the forms $[[\psi](x)],[[\psi](x, y)],[[\psi](x, y, z)],[[\alpha](x)]$, and $[[\alpha](x, y)]$ respectively; and lastly, the functions corresponding to elements of $V_{7}$ will take the value $*$ on all of $U$.

To start, let there be given maps $\phi_{1}$ through $\phi_{6}$ where $\phi_{1}$ maps $V_{1}$ onto $F, \phi_{2}$ maps $V$ one-to-one onto $V_{2}, \phi_{3}$ maps $V \times V$ one-to-one onto $V_{3}, \phi_{4}$ maps $V \times V \times V$ oneto-one onto $V_{4}, \phi_{5}$ maps $V$ one-to-one onto $V_{5}, \phi_{6}$ maps $V \times V$ one-to-one onto $V_{6}$. (The existence of these maps, for arbitrary infinite $U$, is assured by elementary set theory with the axiom of choice.)

Given the sets $V_{0}, \ldots, V_{6}$ and the maps $\phi_{1}, \ldots, \phi_{6}$, we shall now define a map $g: U \times U \rightarrow U$ such that for every $u, x \in U$, the map $G: U \rightarrow U^{U}$ given by $(G(U))(x)$ 
$=g(u, x)$ will be the desired gödelization. Let $C=\bigcup_{i=0}^{5} V_{i}$ and let, $\Lambda_{0}=C \times V$. Then $g_{0}=g \mid \Lambda_{0}$ is given as follows:

$$
\begin{aligned}
g_{0}(u, x) & =\left(\phi_{1}(u)\right)(x) & & \text { if } x \in V_{1} \\
& =\phi_{2}(x) & & \text { if } u=\psi \\
& =\phi_{3}(c, x) & & \text { if } u \in V_{2}, u=\phi_{2}(c) \\
& =\phi_{4}(c, b, x) & & \text { if } u \in V_{3}, u=\phi_{3}(c, b) \\
& =a & & \text { if } u \in V_{4}, u=\phi_{4}(c, b, a) \text { and } x=c \\
& =b & & \text { if } u \in V_{4}, u=\phi_{4}(c, b, a) \text { and } x \neq c \\
& =\phi_{5}(x) & & \text { if } u=\alpha \\
& =\phi_{6}(c, x) & & \text { if } u \in V_{5}, u=\phi_{5}(c)
\end{aligned}
$$

for all $\langle u, x\rangle \in \Lambda_{0}$ and all $c, b, a \in V$.

We now define $\Lambda_{n}$ and $g_{n}$ for all integers $n>0$ as follows:

$\Lambda_{n+1}=\Lambda_{n} \cup\left\{\langle u, x\rangle \mid u=\phi_{6}(p, q)\right.$ where $\langle p, x\rangle,\langle q, x\rangle$

and $g_{n+1}: \Lambda_{n+1} \rightarrow U$ such that and $\left\langle g_{n}(p, x), g_{n}(q, x)\right\rangle$ are all in $\left.\Lambda_{n}\right\}$,

$$
\begin{aligned}
g_{n+1}(u, x) & =g_{n}(u, x) & & \text { if }\langle u, x\rangle \in \Lambda_{n} \\
& =g_{n}\left(g_{n}(p, x), g_{n}(q, x)\right) & & \text { if } u \in \Lambda_{n+1}-\Lambda_{n^{\prime}} \text { and } u=\phi_{6}(p, q) .
\end{aligned}
$$

Now take $g$ to be the function mapping $U \times U \rightarrow U$ such that, for any $u, x \in U$,

$$
\begin{aligned}
g(u, x) & =g_{n}(u, x) & & \text { if there exists } n \text { such that }\langle u, x\rangle \in \Lambda_{n} \\
& =* & & \text { otherwise. }
\end{aligned}
$$

That $g$ is well defined follows from the definition of $g_{n}$. Consider now the structure $\langle U, G\rangle$ where $G$ is the map of $U \rightarrow U^{U}$ such that, for every $u$ and $x$ in $U,(G(u))(x)$ $=g(u, x)$. To see that this structure is indeed a U.R.S., we must show that Axioms 1,2 , and 3 are satisfied.

Let us denote $G(u)$ by $[u]$ for all $u \in U$.

To prove that Axiom 1 is satisfied, we must show that, for every $u \in U,[*](u)$ $=[u](*)=*$. This amounts to showing that $g(*, u)=g(u, *)=*$. Since, for all $\langle x, y\rangle \in U \times U, g(x, y)=*$ if there does not exist $\Lambda_{n}$ such that $\langle x, y\rangle \in \Lambda_{n}$, it suffices to show that for every $n, \Lambda_{n} \subseteq V \times V$. But $\Lambda_{0} \subseteq C \times V$ by definition, and it follows easily by induction on $n$, that for all $n, \Lambda_{n} \subseteq\left(C \cup V_{6}\right) \times V \subseteq V \times V$. Thus Axiom 1 is satisfied.

To prove that Axiom 2 is satisfied, consider the element $\alpha$ of $V_{0}$. Let $p, q$ and $x$ be elements of $V$. Then, from the definition of $g$, we see that

$$
\begin{aligned}
{[[\alpha](p)](q) } & =g(g(\alpha, p), q) \\
& =g\left(\phi_{5}(p), q\right) \\
& =\phi_{6}(p, q) \in V_{6} \subset V,
\end{aligned}
$$

so the first requirement, namely $[[\alpha](p)](q)=*$, is met. 
Second, we see that $[[[\alpha](p)](q)](x)=g(g(g(\alpha, p), q), x)=g\left(\phi_{6}(p, q), x\right)$. Now, in order for Axiom 2 to be satisfied, we must show that

$$
[[[\alpha](p)](q)](x)=[[p](x)]([q](x)) .
$$

We have already shown that the left side equals $g\left(\phi_{6}(p, q), x\right)$. Let us now consider the right side. By definition, $[[p](x)]([q](x))=g(g(p, x), g(q, x))$. Now a necessary condition for this to not equal $*$ is that there exist integers $n, n^{\prime}$ and $n^{\prime \prime}$ such that $\langle p, x\rangle \in \Lambda_{n},\langle q, x\rangle \in \Lambda_{n^{\prime}}$, and $\langle g(p, x), g(q, x)\rangle \in \Lambda_{n^{\prime \prime}}$. If this condition is satisfied, then

$$
\begin{aligned}
{[[p](x)]([q](x)) } & =g_{n^{\prime \prime}}\left(g_{n}(p, x), g_{n^{\prime}}(q, x)\right) \\
& =g_{n^{\wedge}}\left(g_{n^{\wedge}}(p, x), g_{n^{\wedge}}(q, x)\right),
\end{aligned}
$$

where $n^{\wedge}$ is any integer $\geqq n, n^{\prime}, n^{\prime \prime}$. But if this is the case, then, by definition, $\left\langle\phi_{6}(p, q), x\right\rangle \in \Lambda_{n^{\wedge}+1}$ and $[[[\alpha](p)](q)](x)=g\left(\phi_{6}(p, q), x\right)=g_{n^{\wedge}}\left(g_{n^{\wedge}}(p, x), g_{n^{\wedge}}(q, x)\right)$ and thus (1) is satisfied. While on the other hand, if this condition (the existence of $n, n^{\prime}$ and $\left.n^{\prime \prime}\right)$ is not satisfied, then, by definition, for all $n^{\wedge},\left\langle\phi_{6}(p, q), x\right\rangle \notin \Lambda_{n^{\wedge}}$, and $[[[\alpha](p)](q)](x)=*=[[p](x)]([q](x))$, and (1) is again satisfied.

Lastly, to prove that Axiom 3 is satisfied, consider the element $\psi$ of $V_{0}$. Let $c, b, a$, and $x$ be elements of $V$. Then, from the definition of $g_{0}$, we see that

$$
\begin{aligned}
{[[[[\psi](c)](b)](a)](x) } & =g(g(g(g(\psi, c), b), a), x) \\
& =g\left(g\left(g\left(\phi_{2}(c), b\right), a\right), x\right) \\
& =g\left(g\left(\phi_{3}(c, b), a\right), x\right) \\
& =g\left(\phi_{4}(c, b, a), x\right) \\
& = \begin{cases}a & \text { if } x=c \\
b & \text { if } x \neq c\end{cases}
\end{aligned}
$$

which is just what is required for Axiom 3.

Thus all three axioms are satisfied and so $\langle U, G\rangle$ is indeed a U.R.S.

The above argument not only provides a proof for the theorem, but in addition, provides a means for "constructing" a U.R.S. given a 15-tuple $S=\langle\boldsymbol{F}, \alpha, \psi$, $\left.V_{1}, \ldots, V_{6}, \phi_{1}, \ldots, \phi_{6}\right\rangle$. This "construction" will play a central role in the remainder of this paper.

Let $N$ denote the nonnegative integers. We shall now employ the above construction to give (a sketch of) a proof that for any total function $f: N \rightarrow N$ the set of functions recursive in $f$ form (the function set of) a U.R.S. By the set $R(f)$ of functions recursive in $f$, we mean the smallest set of functions which contains the functions

(i) $f$

(ii) $s(n)=n+1$ (the standard successor function)

(iii) $U_{n, i}\left(x_{1}, \ldots, x_{i}, \ldots, x_{n}\right)=x_{i}$ for all $n$ and all $i, 1 \leqq i \leqq n$

(iv) $Z(n)=0$ 
and is closed under the operations of composition, primitive recursion, and minimalization.

THEOREM 5.2. Let $N$ denote the nonnegative integers and let $f: N \rightarrow N$ be a total function. Then there exists a U.R.S. $R_{f}=\langle(N \cup\{*\}), G\rangle$ such that $F\left(R_{f}\right)$ is precisely the set $R(f)$ of functions recursive in $f$ (where the value * is interpreted as " undefined").

Proof. We employ the construction of the preceding theorem to construct $\boldsymbol{R}_{\mathrm{f}}$. Let $s: N \rightarrow N$ be the standard successor function on $N$ (i.e., for every $n \in N$, $s(n)=n+1)$. Then, let $R_{f}$ be the U.R.S. constructed from the 15-tuple, $S=\langle F, \alpha, \psi$, $V_{1}, \ldots, V_{6}, \phi_{1}, \ldots, \phi_{6}>$ where:

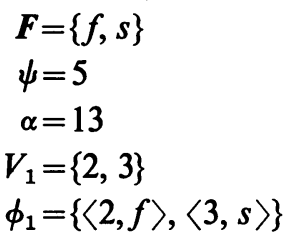

and, for all $x, y, z \in N$,

$$
\begin{aligned}
\phi_{2}(x) & =5^{x+2} \\
\phi_{3}(x, y) & =5^{x+2} \cdot 7^{y+2} \\
\phi_{4}(x, y, z) & =5^{x+2} \cdot 7^{y+2} \cdot 11^{z+2} \\
\phi_{5}(x) & =13^{x+2} \\
\phi_{6}(x, y) & =13^{x+2} \cdot 17^{y+2}
\end{aligned}
$$

and, for $i=2, \ldots, 6, V_{i}=$ range $\left(\phi_{i}\right)$.

That $R_{f}$ is a U.R.S. follows from 5.1. It remains to show that $F\left(R_{f}\right)=R(f)$. We first show that $R(f) \subset F\left(R_{f}\right)$. The functions $f$ and $s$ are in $F\left(R_{f}\right)$ by definition, the functions $U_{n, i}$ are in $F\left(R_{f}\right)$ by 2.2 , and the function $Z$ is the function $\left[k_{0}\right]_{1}$ $=[[\psi](0,0,0)]$ given by 2.1 . Finally, $R_{f}$ is closed under composition by 2.3 , under primitive recursion by 4.3 , and under minimalization by 4.5 . Thus $R(f) \subset F\left(R_{f}\right)$.

To see that $F\left(R_{f}\right) \subset R_{f}$, it clearly suffices to prove that the function $g: N \times N \rightarrow N$ constructed in the proof of 5.1 is recursive in $f$, starting from the above 15-tuple $S$ since this will give us all the 1-ary functions in $F\left(R_{f}\right)$ and that the $n$-ary functions $(n>1)$ are in $R_{f}$ will then follow by composition. The construction is straightforward, but tedious. The essential construction, once $g_{0}$ is known, is given in the proof of the next result. We leave the details to the reader.

We return now to U.R.S. on arbitrary domains. The next lemma provides the foundation for studying the "carrying out within a U.R.S." of the "construction" given in 5.1 .

LEMMA 5.3. Let $I^{\wedge}=\left\langle U, G^{\wedge}\right\rangle$ be a U.R.S. constructed from $S^{\wedge}=\left\langle F^{\wedge}, \alpha^{\wedge}, \psi^{\wedge}\right.$, $\left.V_{1}^{\wedge}, \ldots, V_{6}^{\wedge}, \phi_{1}^{\wedge}, \ldots, \phi_{6}^{\wedge}\right\rangle$ and, for all $e \in V$, let $[e]^{\wedge}$ denote $G^{\wedge}(e)$. Let $I=\langle U, G\rangle$ be a second U.R.S. where, for all $e \in V,[e]$ denotes $G(e)$. If $I$ is such that, 
(a) I has a computable successor pair $\langle[s], z\rangle$ with successor set $N$;

(b) The sets $\bigcup_{i=0}^{5} V_{i}^{\wedge}$ and $V_{6}^{\wedge}$ are computable in $I$ (recall $\left.V_{0}^{\wedge}=\left\{\alpha^{\wedge}, \psi^{\wedge}\right\}\right)$;

(c) There exist functions $\left[\alpha_{1}^{-1}\right]$ and $\left[\alpha_{2}^{-1}\right]$ in $F(I)$ such that, for all $x, y \in V$,

$$
\begin{aligned}
& {\left[\alpha_{1}^{-1}\right]\left(\left[\left[\alpha^{\wedge}\right]^{\wedge}(x)\right]^{\wedge}(y)\right)=x,} \\
& {\left[\alpha_{2}^{-1}\right]\left(\left[\left[\alpha^{\wedge}\right]^{\wedge}(x)\right]^{\wedge}(y)\right)=y,}
\end{aligned}
$$

(i.e., $F(I)$ contains " inverses" for $\left[\alpha^{\wedge}\right]_{2}^{\wedge}$ );

(d) There exists $\left[t^{0}\right]_{2} \in F(I)$ such that, for all $e, x \in V$,

$$
\begin{aligned}
{\left[t^{0}\right](e, x) } & =[e]^{\wedge}(x) & & \text { if } e \in \bigcup_{i=0}^{5} V_{i} \\
& =1 & & \text { otherwise; }
\end{aligned}
$$

then.there exist functions $[T]_{3}$ and $[U]_{3}$ in $F(I)$ such that, for all $e, x \in V$,

$$
[e]^{\wedge}(x)=[U]\left(e, x, \min _{y \in N}[T](e, x, y)=0\right)
$$

and thus, $F\left(1, I^{\wedge}\right) \subset F(1, I)$.

Proof. Let $\Lambda_{n} \subseteq V \times V$ and $g_{n}: \Lambda_{n} \rightarrow V(n=0,1,2, \ldots)$ be the sets and functions respectively used to "construct" $I^{\wedge}$ in accordance with Theorem 5.1. What we will show is that $F(I)$ contains functions $[T]_{3}$ and $[U]_{3}$ such that, for all $e, x \in V$, and every integer $n \geqq 0$,

(i)

$$
\begin{aligned}
& {[T]\left(e, x,[s]^{n}(z)\right)=0 \text { if }\langle e, x\rangle \in \Lambda_{n} \text { and }[e]^{\wedge}(x) \neq *} \\
& =0 \text { only if }\langle e, x\rangle \in \Lambda_{n} \\
& =1 \text { only if }\langle e, x\rangle \notin \Lambda_{n} \\
& =* \text { only if }[e]^{\wedge}(x)=* \text {; }
\end{aligned}
$$

(ii) $[T]\left(e, x,[s]^{n}(z)\right)=0$ implies that, for all $m, 0 \leqq m \leqq n,[T]\left(e, x,[s]^{m}(z)\right)=0$ or 1 ;

$$
\begin{aligned}
{[U]\left(e, x,[s]^{n}(z)\right) } & =g_{n}(e, x)=[e]^{\wedge}(x) & & \text { if }\langle e, x\rangle \in \Lambda_{n} \\
& =* & & \text { only if }[e]^{\wedge}(x)=* .
\end{aligned}
$$

That the desired result (A) will follow from these three properties is an immediate consequence of 4.5. The function $[T]_{3}$ and $[U]_{3}$ are defined jointly by recursion with respect to the successor set $N$ of $\langle[s], z\rangle$. We take:

$$
\begin{aligned}
{[T]\left(e, x,[s]^{0}(z)\right) } & =0 \quad \text { if }\langle e, x\rangle \in \Lambda_{0}=\left(\bigcup_{i=0}^{5} V_{i}\right) \times V \\
& =1 \quad \text { if }\langle e, x\rangle \notin \Lambda_{0}, \\
{[U]\left(e, x,[s]^{0}(z)\right) } & =\left[t^{0}\right](e, x)
\end{aligned}
$$

and for $n \geqq 0$,

$$
\begin{aligned}
{[T]\left(e, x,[s]^{n+1}(z)\right)=} & \left([T]\left(e, x,[s]^{n}(z)\right)=0\right) \\
& \vee\left(\left([T]\left(e, x,[s]^{n}(z)\right)=1\right)\right. \\
& \wedge\left(e \in V_{6}\right) \wedge\left([T]\left(\left[\alpha_{1}^{-1}\right](e), x,[s]^{n}(z)\right)=0\right) \\
& \wedge\left([T]\left(\left[\alpha_{2}^{-1}\right](e), x,[s]^{n}(z)\right)=0\right) \\
& \wedge\left([ T ] \left([U]\left(\left[\alpha_{1}^{-1}\right](e), x,[s]^{n}(z)\right),\right.\right.
\end{aligned}
$$

$\left.\left.\left.[U]\left(\left[\alpha_{2}^{-1}\right](e), x,[s]^{n}(z)\right),[s]^{n}(z)\right)=0\right)\right)$ 


$$
\begin{aligned}
{[U]\left(e, x,[s]^{n+1}(z)\right)=} & {[U]\left(e, x,[s]^{n}(z)\right) \quad \text { if }[T]\left(e, x,[s]^{n}(z)\right)=0 } \\
= & {[U]\left([U]\left(\left[\alpha_{1}^{-1}\right](e), x,[s]^{n}(z)\right),[U]\left(\left[\alpha_{2}^{-1}\right](e), x,[s]^{n}(z)\right),[s]^{n}(z)\right) } \\
& \quad \text { if }[T]\left(e, x,[s]^{n}(z)\right)=1 \quad \text { and }[T]\left(e, x,[s]^{n+1}(z)\right)=0 \\
= & 0 \quad \text { if }[T]\left(e, x,[s]^{n}(z)\right)=[T]\left(e, x,[s]^{n+1}(z)\right)=1 \\
= & * \quad \text { otherwise. }
\end{aligned}
$$

That these functions are well defined and in $F(I)$ follows from 4.3 (and the remarks following it) and from 4.1 and 2.5. Note particularly that the terms in the definition of $[T]_{3}$ are ordered so as to take advantage of the fact that $0 \vee *=0$ and $1 \wedge *=1$; and that the conditions are ordered in the definition of $[U]_{3}$ so that the value $*$ will be taken only if none of the other conditions are met (see 4.1).

To prove that $[T]_{3}$ and $[U]_{3}$ have the three claimed properties, we proceed by induction on $n$. It is clear from the definitions of the functions that the properties all hold for $n=0$; assume now that they hold for $n=k \geqq 0$. To see that they hold for $k+1$, we investigate the properties one at a time.

Consider the first property. From the definition of $\Lambda_{k+1}$ (see proof of 5.1), we know that $\langle e, x\rangle \in \Lambda_{k+1}$ if and only if

$$
\begin{gathered}
\left(\langle e, x\rangle \in \Lambda_{k}\right) \text { or }\left(\left(\langle e, x\rangle \notin \Lambda_{k}\right) \text { and }\left(e \in V_{6}\right)\right. \text { and } \\
\left.\left(\left\langle\left[\alpha_{1}^{-1}\right](e), x\right\rangle \in \Lambda_{k}\right) \text { and }\left(\left\langle\left[\alpha_{2}^{-1}\right](e), x\right\rangle \in \Lambda_{k}\right\rangle\right) \\
\text { and } \left.\left(\left\langle\left[\left[\alpha_{1}^{-1}\right](e)\right]^{\wedge}(x),\left[\left[\alpha_{2}^{-1}\right](e)\right]^{\wedge}(x)\right\rangle \in \Lambda_{k}\right)\right) .
\end{gathered}
$$

Now say $\langle e, x\rangle \in \Lambda_{k+1}$ and $[e]^{\wedge}(x) \neq *$. Then, by the induction hypothesis (1) corresponds directly to the conditions for $[T]\left(e, x,[s]^{k+1}(z)\right)=0$ in the recursive definition of $[T]$ (note that if any of the terms in these conditions took the value $*$, then this would imply $[e]^{\wedge}(x)=*$ by the induction hypothesis and the definition of $[e]^{\wedge}($ see 5.1$\left.)\right)$.

Conversely, if $[T]\left(e, x,[s]^{k+1}(z)\right)=0$, then, by essentially the reverse of the above argument, we see that the conditions in the definition of $[T]_{3}$ imply that the above predicate (1) is satisfied and thus that $\langle e, x\rangle \in \Lambda_{k+1}$; hence, $[T]\left(e, x,[s]^{k+1}(z)\right)=0$ only if $\langle e, x\rangle \in \Lambda_{k+1}$.

Now say that $[T]\left(e, x,[s]^{k+1}(z)\right)=1$. Then, by the definition of $[T]_{3}$ we have:

$$
\begin{aligned}
\left([T]\left(e, x,[s]^{k}(z)\right)=1\right) & \wedge\left(\left(e \notin V_{6}\right) \vee\left([T]\left(\left[\alpha_{1}^{-1}\right](e), x,[s]^{k}(z)\right)=1\right)\right. \\
& \vee\left([T]\left(\left[\alpha_{2}^{-1}\right](e), x,[s]^{k}(z)\right)=1\right) \\
& \vee\left([ T ] \left([U]\left(\left[\alpha_{1}^{-1}\right](e), x,[s]^{k}(z)\right),\right.\right. \\
& \left.\left.\left.\quad[U]\left(\left[\alpha_{2}^{-1}\right](e), x,[s]^{k}(z)\right),[s]^{k}(z)\right)=1\right)\right) .
\end{aligned}
$$

But this, by the induction hypothesis, gives us the negation of the above predicate (1) and thus implies $\langle e, x\rangle \in \Lambda_{k+1}$.

Finally, say $[T]\left(e, x,[s]^{k+1}(z)\right)=*$. Then, it follows from the definition of $[T]_{3}$, that $[T]\left(e, x,[s]^{k}(z)\right) \neq 0$. Now if $[T]\left(e, x,[s]^{k}(z)\right)=*$, then, by the induction 
hypothesis, $[e]^{\wedge}(x)=*$ and we are done. Thus, let us assume that $[T]\left(e, x,[s]^{k}(z)\right)=1$. Now, to avoid $[T]\left(e, x,[s]^{k+1}(z)\right)=0$, we must not have

$$
\begin{aligned}
\left(e \in V_{6}\right) & \wedge\left([T]\left(\left[\alpha_{1}^{-1}\right](e), x,[s]^{k}(z)\right)=0\right) \\
& \vee\left([T]\left(\left[\alpha_{2}^{-1}\right](e), x,[s]^{k}(z)\right)=0\right) \\
& \vee\left([ T ] \left([U]\left(\left[\alpha_{1}^{-1}\right](e), x,[s]^{k}(z)\right),\right.\right. \\
& \left.\left.\quad[U]\left(\left[\alpha_{2}^{-1}\right](e), x,[s]^{k}(z)\right),[s]^{k}(z)\right)=0\right) .
\end{aligned}
$$

But if $e \notin V_{6}$, then $[e]^{\wedge}(x)=*$ (since $[T]\left(e, x,[s]^{k}(z)\right)=1$ implies $\left.e \notin \bigcup_{i=0}^{5} V_{i}\right)$ and we are done. Thus, let us assume $e \in V_{6}$. But then, to avoid $[T]\left(e, x,[s]^{k+1}(z)\right)=1$, at least one of the remaining terms in (2) above must take the value $*$. Then, by the induction hypothesis, it follows that either $\left[\left[\alpha_{1}^{-1}\right](e)\right]^{\wedge}(x)=*,\left[\left[\alpha_{2}^{-1}\right](e)\right]^{\wedge}(x)=*$, or $\left[\left[\left[\alpha_{1}^{-1}\right](e)\right]^{\wedge}(x)\right]^{\wedge}\left(\left[\left[\alpha_{2}^{-1}\right](e)\right]^{\wedge}(x)\right)=*$. Thus, by the definition of $[e]^{\wedge}(5.1)$, we get $[e]^{\wedge}(x)=*$, and so $[T]\left(e, x,[s]^{k}(z)\right)=*$ only if $[e]^{\wedge}(x)=*$.

This completes the inductive step for the first property. As for the second property, clearly, for any $m \geqq 0,[T]\left(e, x,[s]^{m}(z)\right)=*$ implies $[T]\left(e, x,[s]^{m+1}(z)\right)=*$, (see definition of $[T]_{3}$ ) and the property follows immediately.

It remains to establish the third property. Assume first that $\langle e, x\rangle \in \Lambda_{k+1}$ and $[e]^{\wedge}(x) \neq *$. Then by the inductive step for property 1 we know $[T]\left(e, x,[s]^{k+1}(z)\right)=0$. If, in addition $\langle e, x\rangle \in \Lambda_{k}$, then, by the induction hypothesis, $[T]\left(e, x,[s]^{k}(z)\right)=0$ and so, by the definition of $[U]_{3},[U]\left(e, x,[s]^{k+1}(z)\right)=[U]\left(e, x,[s]^{k}(z)\right)$ which, by the induction hypothesis, $=[e]^{\wedge}(x)$ as desired. But if $\langle e, x\rangle \notin \Lambda_{k}$ then, since $[e]^{\wedge}(x) \neq *$, we have $[T]\left(e, x,[s]^{k}(z)\right)=1$ and so, by the definition of $[U]_{3}$,

$$
[U]\left(e, x,[s]^{k+1}(z)\right)=[U]\left([U]\left(\left[\alpha_{1}^{-1}\right](e), x,[s]^{k}(z)\right),[U]\left(\left[\alpha_{2}^{-1}\right](e), x,[s]^{k}(z)\right),[s]^{k}(z)\right)
$$

which, by the induction hypothesis $=\left[\left[\left[\alpha_{1}^{-1}\right](e)\right]^{\wedge}(x)\right]^{\wedge}\left(\left[\left[\alpha_{2}^{-1}\right](e)\right]^{\wedge}(x)\right)=[e]^{\wedge}(x)$ by the definition of $[e]^{\wedge}(5.1)$.

Next, consider the case where $\langle e, x\rangle \in \Lambda_{k+1}$, but $[e]^{\wedge}(x)=*$. Then from the inductive step for property 1 , it follows that $[T]\left(e, x,[s]^{k+1}(z)\right)=0$ or $*$. In the latter case, it follows, from the definition of $[U]_{3}$, that $[U]\left(e, x,[s]^{k+1}(z)\right)=*$ so assume $[T]\left(e, x,[s]^{k+1}(z)\right)=0$. But then, from property 2 we have $[T]\left(e, x,[s]^{k}(z)\right)$ $=0$ or 1 and we get $[T]\left(e, x,[s]^{k+1}(z)\right)=[e]^{\wedge}(x)$ by essentially the same argument as for the $[e]^{\wedge}(x) \neq *$ case. Thus, we have that $\langle e, x\rangle \in \Lambda_{k+1}$ implies $[U]\left(e, x,[s]^{k+1}(z)\right)$ $=[e]^{\wedge}(x)$.

Finally, say $[U]\left(e, x,[s]^{k+1}(z)\right)=*$. Then, from the definition of $[U]$ it follows that $[T]\left(e, x,[s]^{k}(z)\right)=*$ or $[T]\left(e, x,[s]^{k+1}(z)\right)=*$ or $[U]\left([U]\left(\left[\alpha_{1}^{-1}\right](e), x,[s]^{k}(z)\right)\right.$, $[U]\left(\left[\alpha_{2}^{-1}\right](e), x,[s]^{k}(z)\right)=*$. But, by the induction hypothesis and the inductive step for property 1 it follows, from any of these, that $[e]^{\wedge}(x)=*$ and thus, $[U]\left(e, x,[s]^{k+1}(z)\right)=*$ only if $[e]^{\wedge}(x)=*$. This then completes the inductive step for the third property and so, by induction, the three properties hold for all $n \geqq 0$.

That, for all $e, x \in V$,

$$
[e]^{\wedge}(x)=[U]\left(e, x, \min _{y \in N}[T](e, x, y)=0\right)
$$


then follows immediately from the three properties. Note particularly that the second property insures that the min $y \in N$ will be "found" if it exists. That this function is in $F(I)$ follows from 2.3 and it follows immediately from this result that $F\left(1, I^{\wedge}\right) \subset F(I)$.

The preceding result shows that under the given conditions we can "construct" the set $F\left(1, I^{\wedge}\right)$ of all 1-ary functions of $I^{\wedge}$ "within" the U.R.S. $I$ in a uniform manner (i.e., just using $[T]_{3}$ and $[U]_{3}$ ). This immediately suggests the study of the case where $I=I^{\wedge}$. In addition, the functions $[T]_{3}$ and $[U]_{3}$ are strongly reminiscent of those employed in the Kleene Normal Form theorem of partial recursive function theory, except that they are not necessarily $*$-total. This suggests a strengthening of the above result to the case where $I=I^{\wedge}$ and $[T]_{3}$ and $[U]_{3}$ are *-total in order to produce U.R.S. with a normal form theorem. The following definition provides a class of U.R.S. with these properties.

A U.R.S. $I=\langle U, G\rangle$ will be said to be highly constructible if there exists a 15tuple $S=\left\langle\boldsymbol{F}, \alpha, \psi, V_{1}, \ldots, V_{6}, \phi_{1}, \ldots, \phi_{6}\right\rangle$ such that $I$ is constructible from $S$ and

(1) Every function in $\boldsymbol{F}$ is $*$-total;

(2) There exists a computable successor pair $\langle[s], z\rangle$ in $I$;

(3) The predicates " $x \in V_{i}$ ", $i=1,2, \ldots, 6$ are computable in $I$;

(4) There exist $*$-total functions $\left[\alpha_{1}^{-1}\right]$ and $\left[\alpha_{2}^{-1}\right]$ in $F(I)$ such that, for every $p$ and $q$ in $V$,

$$
\left[\alpha_{1}^{-1}\right]([\alpha](p, q))=p, \quad\left[\alpha_{2}^{-1}\right]([\alpha](p, q))=q .
$$

THEOREM 5.4. Let $N$ denote the nonnegative integers and let $f: N \rightarrow N$ be a total function. Then there exists a highly constructible U.R.S. $R_{f}=\langle(N \cup\{*\}), G\rangle$ such that $F\left(R_{f}\right)$ is precisely the set $R(f)$ of functions recursive in $f$.

Proof. This result is an almost immediate corollary of the proof of 5.2. All that remains to be done is to show that the U.R.S. $R_{f}$ given there is highly constructible, but this is evident on the basis of very elementary results of the theory of partial recursive functions.

TheORem 5.5 (Normal Form TheORem). Let $I=\langle U, G\rangle$ be a highly constructible $U . R . S$. and let $\langle[s], z\rangle$ be a computable successor pair in I with successor set $N$. Then there exists a $*$-total function $[U]_{3}$ and a 3-ary computable predicate $[T]_{3}$ in $F(I)$ such that, for all $e$ and $x$ in $V$,

$$
[e](x)=[U]\left(e, x, \min _{y \in N} T(e, x, y)=0\right) .
$$

Proof. Let $[T]_{3}$ and $[U]_{3}$ be the functions developed in Lemma 5.3. All that remains to be shown is that these functions are *-total. We know that for all $e, x \in V$ and $y \notin N,[T](e, x, y),[U](e, x, y) \neq *$. Thus $[T]_{3}$ and $[U]_{3}$ are not $*$-total only if there exists a triple $\langle e, x, n\rangle, e, x \in V, n$ an integer $\geqq 0$, such that

$$
\left([T]\left(e, x,[s]^{n}(z)\right)=*\right) \vee\left([U]\left(e, x,[s]^{n}(z)\right)=*\right) .
$$


Consider the set of all such triples. If this set is not empty, then there will exist a least integer $n$ for which there exist $e$ and $x$ in $V$ satisfying (1). Now for all $e, x \in V$, $[T]\left(e, x,[s]^{0}(z)\right) \neq *$ and, since all the elements of $\boldsymbol{F}$ are *-total, we have $\langle e, x\rangle \in \Lambda_{0}$ implies $[e](x) \neq *$. Thus, from the definition of $[U]_{3}$, we have $[U]\left(e, x,[s]^{0}(x)\right) \neq *$ for all $e, x \in V$. Hence, the minimal $n$ must be greater than 0 . But then, from inspection of the recursive step in the definitions of $[T]_{3}$ and $[U]_{3}$ (proof of 5.3), we see that (1) above cannot be satisfied without contradicting the minimality of $n$ since the values of $[T]\left(e, x,[s]^{n}(z)\right)$ and $[U]\left(e, x,[s]^{n}(z)\right)$ are then given in terms of their values on $[s]^{n-1}(z)$, for which, by the minimality of $n,[T]_{3}$ and $[U]_{3}$ are $*$-total. Thus, $[T]_{3}$ and $[U]_{3}$ are $*$-total.

To complete this section, we prove a number of results for highly constructible U.R.S. which, as we will later show, are not true for arbitrary U.R.S. In particular, we shall show that the usual proof of the existence of recursively inseparable semicomputable (i.e., recursively enumerable sets) holds for highly constructible U.R.S. We first give a result (which does not hold for arbitrary U.R.S.) which shows that recursive inseparability does not arise trivially in highly constructible U.R.S.

THEOREM 5.6. If I is a highly constructible U.R.S. and $P(x)$ is a predicate such that both $P(x)$ and $\sim P(x)$ are semicomputable in $I$, then $P(x)$ is computable in $I$.

Proof. If $P(x)$ and $\sim P(x)$ are both semicomputable in $I$, then, by definition, there exist $p, q \in V$ such that $[p]_{1}$ and $[q]_{1}$ are the semicharacteristic functions of $P(x)$ and $\sim P(x)$ respectively. Now, by 5.5 and 2.5 there clearly exists a function $[g]_{2} \in F(I)$ such that for all $x \in V$ and all integers $n \geqq 0$

$$
\begin{aligned}
{[g]\left(x,[s]^{n}(z)\right) } & =0 & & \text { if }[T]\left(p, x,[s]^{n}(z)\right)=0 \\
& =1 & & \text { if }[T]\left(q, x,[s]^{n}(z)\right)=0 .
\end{aligned}
$$

But then there exists $\rho \in V$ such that, for every $x \in V$,

$$
[\rho](x)=[g]\left(x, \min _{y \in N}([T](p, x, y)=0 \text { or }[T](q, x, y)=0)\right)
$$

by virtue of 4.1 and 4.5 . But clearly, $[\rho]_{1}$ is the characteristic function of $P(x)$ and thus $P(x)$ is computable in $I$.

We say that two disjoint sets $A$ and $B$ in a U.R.S. $I$ are recursively inseparable in $I$ if there does not exist a computable set $C$ in $I$ such that $A \subset C$ and $B \subset V-C$. To prove that there exist recursively inseparable semicomputable sets in highly constructible U.R.S., we shall use essentially the same proof as used by Smullyan [5] to establish this result for the partial recursive functions. We need the following simple notion and result:

Given two semicomputable sets $\omega_{u}$ and $\omega_{v}$ in a highly constructible U.R.S. I, we say that, $x \in \omega_{u}$ before $x \in \omega_{v}$ if and only if $(\exists n)\left([T]\left(u, x,[s]^{n}(z)\right)=0\right.$ and $\left.(\forall m \leqq n)\left([T]\left(v, x,[s]^{m}(z)\right)=1\right)\right)$. Let

$$
\begin{aligned}
& \omega_{u}^{\prime}=\left\{x \mid x \in \omega_{u} \text { before } x \in \omega_{v}\right\} \\
& \omega_{v}^{\prime}=\left\{x \mid x \in \omega_{v} \text { before } x \in \omega_{u}\right\} .
\end{aligned}
$$


LEMMA 5.7. If I is a highly constructible U.R.S., then for every $u, v \in V$, we have that $\omega_{u}^{\prime}$ and $\omega_{v}^{\prime}$ are semicomputable and disjoint and they are respective supersets of $\left(\omega_{u}-\omega_{v}\right)$ and $\left(\omega_{v}-\omega_{u}\right)$.

Proof. To see that the sets are semicomputable, consider ' $\omega_{u}^{\prime}$. By 2.3 there exists $[r] \in F(1, I)$ such that, for every $x \in V$,

$$
[r](x)=[T]\left(v, x, \min _{y \in N}([T](u, x, y)=0)\right) .
$$

But from the proof of 5.3, we see that if $[T]\left(v, x,[s]^{n}(z)\right)=0$, then for all $m \geqq n$, $[T]\left(v, x,[s]^{m}(z)\right)=0$. Thus, for every $x \in V$,

$$
\begin{aligned}
{[r](x) } & =1 & & \text { if } x \in \omega_{u}^{\prime} \\
& =* & & \text { otherwise }
\end{aligned}
$$

and so $\left[[\gamma]\left(k_{0}, r\right)\right]=\left[k_{0}\right] \cdot[r]$ is the semicharacteristic function of $\omega_{u}^{\prime}$. Similarl $\mathrm{y}, \omega_{v}^{\prime}$ is semicomputable. The remaining claims are obviously true.

THEOREM 5.8. If I is a highly constructible U.R.S., then there exist disjoint semicomputable sets in I which are not recursively separable.

Proof. We shall construct semicomputable sets $P=\omega_{p}$ and $Q=\omega_{q}$ and show that the (not necessarily semicomputable sets) $(P-Q)$ and $(Q-P)$ are recursively inseparable. But from this, it will follow immediately that the above defined semicomputable supersets $\omega_{p}^{\prime}$ and $\omega_{q}^{\prime}$ of, respectively, $(P-Q)$ and $(Q-P)$, are recursively inseparable.

Let $c$ be a fixed element of $V$ and let $[t]_{2} \in F(I)$ such that, for all $x, y \in V$, $[t](x, y)=[\psi](c, x, y)$. From the fact that range $\left([\phi]_{3}\right)$ is computable, it is easily shown that range $\left([t]_{2}\right)$ is computable and that there exist $*$-total functions $\left[\lambda_{1}\right]_{1}$ and $\left[\lambda_{2}\right]_{1}$ in $F(I)$ such that for all $x_{1}, x_{2} \in V$ and $i=1,2,\left[\lambda_{i}\right]\left([t]\left(x_{1}, x_{2}\right)\right)=x_{i}$, and for all $w \notin$ range $\left([t]_{2}\right),\left[\lambda_{i}\right](w)=0$. Now define the sets $P$ and $Q$ by

$$
x \in P \Leftrightarrow x \in \omega_{\lambda_{1}}(x), \quad x \in Q \Leftrightarrow x \in \omega_{\lambda_{2}}(x) .
$$

To see that $P$ is semicomputable, consider the function $[p]$ of $F(I)$, given by 2.3, such that for every $x \in V,[p](x)=\left[k_{0}\right]\left(\min _{y \in N}[T]\left(\left[\lambda_{1}\right](x), x, y\right)=0\right)$. Clearly, $[p]_{1}$ is the semicharacteristic function of $P$. A similar proof gives us a function $[q]$ for $Q$.

Our claim now is that there does not exist a computable set $B$ such that $(P-Q) \subset V-B$. For assume such a $B$ existed and let $[u]$ be the semicharacteristic function of $B$ and let $v$ be the semicharacteristic function of $V-B$. Now consider $w=[t](v, u)$

(1) $w \in P \Leftrightarrow w \in \omega_{v}=V-B$ by definition $P$

(2) $w \in Q \Leftrightarrow w \in \omega_{u}=B$ by definition $Q$.

From which it follows that

(3) $w \in\left(\omega_{v}-\omega_{u}\right)=((V-B)-B)=V-B \Leftrightarrow w \in(P-Q) \Rightarrow w \in B$

(4) $w \in\left(\omega_{u}-\omega_{v}\right)=(B-(V-B))=B \Leftrightarrow w \in(Q-P) \Rightarrow w \in V-B$. 
Hence, $x \in B \Leftrightarrow x \in V-B$, a contradiction. Thus, no such computable set $B$ can exist which separates $(P-Q)$ and $(Q-P)$, and clearly then, $\omega_{p}^{\prime}$ and $\omega_{q}^{\prime}$ must be likewise recursively inseparable. Since $\omega_{p}^{\prime}$ and $\omega_{q}^{\prime}$ are semicomputable sets, by 5.7, this then completes the proof.

The following results which we state but do not prove will be useful in the next section.

LEMMA 5.9. Let $I=\langle U, G\rangle$ be a highly constructible U.R.S. where $\phi \in V$ satisfying Axiom 3 for $I$. Then

(1) The functions $[\psi]_{1},[\psi]_{2}$ and $[\psi]_{3}$ are one-to-one on the sets $V, V \times V$ and $\{x, y, z \mid x, y, z \in V$ and $y \neq z\}$ respectively.

(2) The sets $\{\psi\}$, range $\left(\left[\psi_{1}\right)\right.$, range $\left(\left[\psi_{2}\right)\right.$ and range $\left(\left[\psi_{3}\right]_{3}\right)$ are pairwise disjoint.

(3) If range $\left([\psi]_{2}\right)$ and range $\left([\psi]_{3}\right)$ are computable, then there exist $\mu_{1}, \mu_{2}, \mu_{3} \in V$ such that, for all $w, x \in V$

$$
\begin{array}{rlrl}
{\left[\mu_{1}\right](w, x)} & =y & & \text { if } x=[\psi](w, y) ; y \in V \\
=1 & & \text { otherwise } \\
{\left[\mu_{2}\right](w, x)=y} & & \text { if } x=[\psi](w, y, z) ; y, z \in V \\
=1 & & \text { otherwise } \\
{\left[\mu_{3}\right](w, x)=z} & \text { if } x=[\psi](w, y, z) ; y, z \in V \\
=1 & & \text { otherwise. }
\end{array}
$$

(4) If range $\left(\left[\mathrm{H}_{2}\right)\right.$ and range $\left([\psi]_{3}\right)$ are computable, then for every integer $n>1$, there exists a function $\left[p_{n}\right] \in F(n, I)$ and functions $\left[\Pi_{1}^{n}\right], \ldots,\left[\Pi_{n}^{n}\right] \in F(1, I)$ such that for every $n$-tuple $x^{(n)}=\left\langle x_{1}, x_{2}, \ldots, x_{n}\right\rangle \in V^{n}$,

(i) $\left[p_{n}\right]\left(x^{(n)}\right) \in V$,

(ii) for $i=1,2, \ldots, n,\left[\Pi_{i}^{n}\right]\left(\left[p_{n}\right]\left(x^{(n)}\right)\right)=x_{i}$,

(iii) range $\left(\left[p_{n}\right]_{n}\right)$ is computable.

Proof. The proofs are simple but tedious. We leave them to the reader.

6. Intrinsic relative computability-the U.R.S. lattice. Given two U.R.S. I and $I^{\prime}$ with domain $U$, we say that $I^{\prime}$ is an extension of $I$ if $F(I) \subseteq F\left(I^{\prime}\right)$. What we wish to show in this section is that if we consider the set of all U.R.S. which are extensions of a given highly constructible U.R.S., then the extensionality relationship on this set induces a relationship on the set of *-admissible functions which is a natural generalization of the "computable in" relation. Indeed, where the given highly constructible U.R.S. is the set of partial recursive functions, then the induced relationship on the set of total functions is precisely the "recursive in" relationship (or Turing reducibility relationship) of the traditional theory of computability. Thus, the extension relation, which is intrinsic to the theory of U.R.S., provides an intrinsic notion of relative computability.

The following lemma, while of little interest in its own right, will be very useful. 
Lemma 6.1. Let $I=\langle U, G\rangle$ and $I^{\prime}=\left\langle U, G^{\prime}\right\rangle$ be two U.R.S. with domain U. Then $\left(F(1, I) \cup\left\{[\psi]_{3}\right\}\right) \subseteq F\left(I^{\prime}\right)$ implies $I^{\prime}$ is an extension of $I\left(\right.$ i.e., $\left.F(I) \subseteq F\left(I^{\prime}\right)\right)$.

Proof. Let the argument be the same as in the proof of 3.5 up through the definition of the function $\left[\lambda_{2}\right]$. Then to complete the proof, we must show that $F(2, I)$ $\subseteq F\left(2, I^{\prime}\right)$. This may be done as follows:

Let $[r]_{2} \in F(2, I)$. By 2.3 there must exist $[w]_{1} \in F(I)$ such that, for all $z \in V$, $[w](z)=[r]\left(\left[\lambda_{1}\right](z),\left[\lambda_{2}\right](z)\right)$. But then, since $F(1, I) \subset F\left(1, I^{\prime}\right)$, there must exist $\left[w^{\prime}\right]_{1}^{\prime} \in F\left(I^{\prime}\right)$ such that $\left[w^{\prime}\right]_{1}^{\prime}=[w]_{1}$. Thus, since we have assumed $[\psi]_{3} \in F\left(I^{\prime}\right)$, it follows, by 2.3 , that there exists $\left[r^{\prime}\right]_{2}^{\prime} \in F\left(I^{\prime}\right)$ such that, for all $x, y \in V$,

$$
\left[r^{\prime}\right]^{\prime}(x, y)=\left[w^{\prime}\right]^{\prime}([\psi](c, x, y))
$$

which, by the above,

$$
\begin{aligned}
& =[w]([\psi](c, x, y)) \\
& =[r]\left(\left[\lambda_{1}\right]([\psi](c, x, y)),\left[\lambda_{2}\right]([\psi](c, x, y))\right) \\
& =[r](x, y),
\end{aligned}
$$

whence $[r]_{2}=\left[r^{\prime}\right]_{2}^{\prime} \in F(2, I)$ and so $F(2, I) \subset F\left(2, I^{\prime}\right)$ and thus $F(I) \subseteq F\left(I^{\prime}\right)$ by the same argument as in 3.5.

In the development of "relative computability" in this section, we shall always start from a highly constructible U.R.S. and consider its extensions. This seeming restriction is justified since the U.R.S. of partial recursive functions is highly constructible. However, inasmuch as the reader may be interested in applications other than to the partial recursive functions, we note the following. By 5.1 we know that there exist highly constructible U.R.S. on all infinite domains and thus the results of this section are applicable to all domains. On the other hand, it can be shown that there are U.R.S. on every domain which are not highly constructible (see 6.12). However, as the following result shows, we can always replace a U.R.S. by a highly constructible extension.

THEOREM 6.2. Every U.R.S. has an extension which is highly constructible.

Proof. Let $[\delta]$ be the diagonal function of $I$ (see 3.2) and let $[\psi]_{2}$ be the function given by Axiom 3 for $I$. Let $0 \in V$-range $\left(\left[\psi_{\psi}\right]_{2}\right)$, and let $f: U \rightarrow U$ be the function such that, for all $x \in V$,

$$
\begin{aligned}
f(x) & =[\psi](0,[\delta](x)) & & \text { if }[\delta](x) \neq * \\
& =0 & & \text { otherwise. }
\end{aligned}
$$

While $f \notin F(I)$, we see immediately that it is *-total and that, for all $x, y \in V$, $f(x)=f(y) \Leftrightarrow[\delta](x)=[\delta](y)$. Now, since $[\psi]_{2}$ is one-to-one, there exists a function $h: U \rightarrow U$ such that, for every $x \in V$,

$$
\begin{aligned}
h(x)=y & \text { if } x=[\psi](0, y) \\
=0 & \text { if } x \notin \operatorname{range}\left([[\psi](0)]_{1}\right) .
\end{aligned}
$$


Again, $h$ is not necessarily in $F(I)$, but it is $*$-total. Now pick $V_{1}, \ldots, V_{6}$ and $\phi_{1}, \ldots, \phi_{6}$ with $\operatorname{Card}\left(V_{1}\right)=\operatorname{Card}(U)$ in accordance with 5.1, and let $g: U \rightarrow U$ such that for every $x \in V$,

$$
\begin{aligned}
g(x) & =[\psi](z, b, c) & & \text { if } x=\phi_{4}(a, b, c) \in V_{4} \\
& =0 & & \text { if } x \notin V_{4} .
\end{aligned}
$$

Then, let $I^{\wedge}=\left\langle U, G^{\wedge}\right\rangle$ be the highly constructible U.R.S. constructed from the 15-tuple $S=\left\langle\left(F^{v}(I) \cup\{f, h, g\}\right), \alpha^{\wedge}, \psi^{\wedge}, V_{1}, \ldots, V_{6}, \phi_{1}, \ldots, \phi_{6}\right\rangle$ (note: $\phi_{1}$ must, of course, be chosen after $g$ is defined). Let $[u]^{\wedge}$ denote $G^{\wedge}(u)$ for all $u \in U$. Since $f, h, g \in F\left(1, I^{\wedge}\right)$, it is easy to see that there must exist $\lambda \in V$ such that for all $x \in V$

$$
\begin{array}{rlrl}
{[\lambda]^{\wedge}(x)} & =h(f(x)) & & \text { if } f(x) \neq 0 \\
& =* & & \text { otherwise } \\
& =[\delta](x) . &
\end{array}
$$

Thus, $[\lambda]_{1}^{\wedge}=[\delta]$ and so $\{[\delta]\} \cup F^{v}(I) \subseteq F\left(I^{\wedge}\right)$. But, by 3.2 every 1-ary function $[u]$

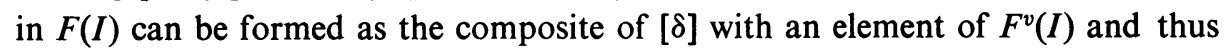
$F(1, I) \subseteq F\left(1, I^{\wedge}\right)$. On the other hand, $g, \phi_{4} \in F\left(I^{\wedge}\right)$ (recall $\left[\psi^{\wedge}\right]_{3}^{\wedge}=\phi_{4}$ by 5.1), Thus, there must exist $\tau \in V$ such that for all $x, y, z \in V$

$$
[\tau]^{\wedge}(x, y, z)=g\left(\phi_{4}(x, y, z)\right)=[\psi](x, y, z)
$$

Thus $[\psi]_{3} \in F\left(I^{\wedge}\right)$ and so, by $6.1, F(I) \subset F\left(I^{\wedge}\right)$.

In proving results concerning the extension-relation, we shall make considerable use of the "construction" of Theorem 5.1. The following lemma establishes conditions under which this "construction" can be used to form extensions. First, a definition:

Given a U.R.S. $I$ and a set $A$ of $*$-admissible functions, we say that a second U.R.S. $I^{\prime}=\left\langle U, G^{\prime}\right\rangle$ is an $A$-extension of $I$ if $F(I) \cup A \subseteq F\left(I^{\prime}\right)$. If $A$ contains only one element, say $g$, then we will write $g$-extension instead of $\{g\}$-extension.

LEMMA 6.3. Let I be a highly constructible U.R.S. constructed from $S=\langle\boldsymbol{F}, \alpha, \psi$, $V_{1}, \ldots, V_{6}, \phi_{1}, \ldots, \phi_{6}>$ where $V-\bigcup_{i=0}^{6} V_{i} \neq \varnothing$. Let $F^{\prime}$ be any set of 1 -ary $*$-admissible functions such that $\mathrm{Card}\left(\boldsymbol{F}^{\prime}\right) \leqq \mathrm{Card}\left(V-\bigcup_{i=0}^{6} V_{i}\right)$. Then where $\phi^{\prime}$ is a one-toone map of $\boldsymbol{F}^{\prime}$ onto $V^{\prime} \subseteq V-\bigcup_{i=0}^{6} V_{i}$, the U.R.S. $I^{\prime}=\left\langle U, G^{\prime}\right\rangle$ constructed from $S^{\prime}=\left\langle\boldsymbol{F} \cup \boldsymbol{F}^{\prime}, \alpha, \psi, V^{\prime} \cup V_{1}, V_{2}, \ldots, V_{6}, \phi^{\prime} \cup \phi_{1}, \phi_{2}, \ldots, \phi_{6}\right\rangle$ is an $\boldsymbol{F}^{\prime}$-extension of $I$.

Proof. From the construction (5.1), it follows that $F^{\prime} \subseteq F\left(I^{\prime}\right)$. Thus, what we must show is that $F(I) \subseteq F\left(I^{\prime}\right)$. Since the construction also insures that $[\psi]_{3} \in F\left(I^{\prime}\right)$, it remains only to show that $F(1, I) \subseteq F\left(1, I^{\prime}\right)$. Let $\langle[s], z\rangle$ be a computable successor 
pair for $I$ with successor set $N$. Then we know that there exist $*$-total functions $[T]_{3}$ and $[U]_{3}$ in $F(I)$ such that for all $\langle u, x\rangle \in V$,

$$
[u](x)=[U]\left(u, x, \min _{y \in N}[T](u, x, y)=0\right) .
$$

It follows then that we need only show that $[T]_{3},[U]_{3}$, and $[s]_{1} \in F\left(I^{\prime}\right)$.

Given $u \in V$, let $[u]$ denote $G(u)$ and let $[u]^{\prime}$ denote $G^{\prime}(u)$. Let $\Lambda_{i}$ and $\Lambda_{i}^{\prime}$, $i=0,1,2, \ldots$ be the sets employed in Theorem 5.1 to define $G$ and $G^{\prime}$ respectively. By induction on $i$ we see easily that $\Lambda_{i} \subseteq \Lambda_{i}^{\prime}$ for all $i$ and that for all $\langle u, x\rangle \in V$, if $\langle u, x\rangle \in \Lambda_{i}$ then $[u]_{1}^{\prime}=[u]_{1}$. From this it follows, by induction on $n$, that if $[u]_{n}$ is *-total, then $[u]_{n}^{\prime}=[u]_{n}$. Thus, $F^{v}(I) \subseteq F^{v}\left(I^{\prime}\right)$ and, in particular, $[U]_{3},[T]_{3}$, $[s]_{1} \in F^{v}\left(I^{\prime}\right)$. But then, from 5.5 it follows that $F(1, I) \subseteq F\left(1, I^{\prime}\right)$ and so, by 6.1 , that $F(I) \subseteq F\left(I^{\prime}\right)$.

We now show that where we start from an appropriate highly constructible U.R.S. that the "construction" yields a minimal $f$-extension for any $*$-admissible function $f$. The lemma requires that the original U.R.S. $I^{\prime}$ be constructed with sets $V_{1}, \ldots, V_{6}$ such that $V-\bigcup_{i=0}^{6} V_{i} \neq \varnothing$. The three results following the lemma show that this restriction is inessential; that is, such minimal $f$-extensions always exist. Thus, the lemma leads to Theorem 6.7, which states the same result without the above restriction.

LEMMA 6.4. Let $I^{\prime}$ be a highly constructible U.R.S. constructed from $S^{\prime}=\langle\boldsymbol{F}, \alpha, \psi$, $V_{1}, \ldots, V_{6}, \phi_{1}, \ldots, \phi_{6}>$ where $V-\bigcup_{i=1}^{6} V_{i} \neq \varnothing$, and let $f: U \rightarrow U$ be a $*$-admissible function. Then there exists a minimal f-extension $I_{f}$ of $I^{\prime}$ in the sense that if $I$ is any $f$-extension of $I^{\prime}$, then $F\left(I_{f}\right) \subseteq F(I)$.

Proof. Let the highly constructible U.R.S. $I^{\prime}$ be "constructed" from $S^{\prime}=\langle\boldsymbol{F}, \alpha, \psi$, $\left.V_{1}, \ldots, V_{6}, \phi_{1}, \ldots, \phi_{6}\right\rangle$ and assume that $V_{7}=V-\bigcup_{i=0}^{6} V_{\imath} \neq \varnothing$. We claim that the desired $f$-extension $I_{f}$ is the U.R.S. $I_{f}=\left\langle U, G_{f}\right\rangle$ "constructed" from $S^{\wedge}=\left\langle F^{\wedge}, \alpha, \psi\right.$, $\left.V_{1}^{\wedge}, V_{2}, \ldots, V_{6}, \phi_{1}^{\wedge}, \ldots, \phi_{2}, \ldots, \phi_{6}\right\rangle$, where:

$$
\begin{aligned}
\boldsymbol{F}^{\wedge} & =\boldsymbol{F} \cup\{f\} \\
V_{1}^{\wedge} & =V_{1} \cup\left\{v_{f}\right\} ; v_{f} \in V_{7} \\
\phi_{1}^{\wedge} & =\phi_{1} \cup\left\{\left\langle v_{f}, f\right\rangle\right\},
\end{aligned}
$$

and $\alpha, \psi, V_{2}, \ldots, V_{6}, \phi_{2}, \ldots, \phi_{6}$ are as in $S^{\prime}$.

Let $I=\langle U, G\rangle$ be an arbitrary $f$-extension of the highly constructible U.R.S. $I^{\prime}=\langle U, G\rangle$ and, for every $u \in U$, let $[u]$ denote $G(u),[u]^{\prime}$ denote $G^{\prime}(u)$, and $[u]^{\wedge}$ denote $G_{f}(u)$. What we must show then is that $F\left(I^{\wedge}\right) \subset F(I)$. We know that $[\psi]_{3}^{\wedge}=[\psi]_{3}^{\prime} \in F\left(I^{\prime}\right) \subseteq F(I)$. Thus, by 6.1 , all we need to show is that $F\left(1, I^{\wedge}\right) \subseteq F(1, I)$. To prove this we employ 5.3. The conditions of 5.3 may be established as follows.

Condition 1 is satisfied since the computable successor pair $\left\langle[s]^{\prime}, z\right\rangle$ of $I^{\prime}$ is perforce in $F(I)$. Condition 2 is satisfied since the characteristic functions of $V_{2}, \ldots, V_{6}$ are in $F\left(I^{\prime}\right) \subset F(I)$ and the characteristic function of $V_{1}$ can clearly be extended to 
$V_{1} \cup\left\{v_{f}\right\}$ by virtue of 2.4. Similarly, the functions $\left[\alpha_{1}^{-1}\right]^{\prime},\left[\alpha_{2}^{-1}\right]^{\prime} \in F\left(I^{\prime}\right) \subset F(I)$ and $[\alpha]_{2}^{\wedge}=[\alpha]_{2}^{\prime}$ so condition 3 is satisfied. Lastly, since $F\left(I^{\prime}\right) \subset F(I)$ and $f \in F(I)$, there exists, by 2.5 , a function $\left[t^{0}\right]_{2} \in F(I)$ such that, for all $e, x \in V$,

$$
\begin{aligned}
{\left[t^{0}\right](e, x) } & = \begin{cases}{[e]^{\prime}(x)} & \text { if } e \in \bigcup_{i=0}^{s} V_{i} \\
f(x) & \text { if } e=v_{f} \\
0 & \text { otherwise }\end{cases} \\
& = \begin{cases}{[e]^{\wedge}(x)} & \text { if } e \in V_{1}^{\wedge} \cup V_{0} \cup V_{2} \cup \cdots \cup V_{5} \\
0 & \text { otherwise, }\end{cases}
\end{aligned}
$$

and thus the fourth condition is satisfied. Hence, 5.3 applies so $F\left(1, I^{\wedge}\right) \subset F(1, I)$, and so, by $6.1, F\left(I^{\wedge}\right) \subset F(I)$. Thus, since $I$ was an arbitrary $f$-extension of $I^{\prime}$, we have that $F\left(I^{\wedge}\right)$ is minimal.

THEOREM 6.5. A necessary and sufficient condition on a U.R.S. $I=\langle U, G\rangle$ for there to exist a highly constructible U.R.S. $I^{\wedge}$ such that $F\left(I^{\wedge}\right) \subseteq F(I)$ is that I contain a computable successor pair $\langle[s], z\rangle$ and that there is $\psi \in V$ satisfying Axiom 3, and such that the sets range $\left([\psi]_{2}\right)$ and range $\left([\psi]_{3}\right)$ are computable in $I$.

Proof. We shall give the requisite 15-tuple

$$
S^{\wedge}=\left\langle F^{\wedge}, \alpha^{\wedge}, \psi^{\wedge}, V_{1}^{\wedge}, \ldots, V_{6}^{\wedge}, \phi_{1}^{\wedge}, \ldots, \phi_{6}^{\wedge}\right\rangle
$$

for $I^{\wedge}$. Then show that $I^{\wedge}$ is highly constructible, and lastly, we shall show that $F\left(I^{\wedge}\right) \subset F(I)$.

Let $0,1,2,3,4,5$, and 6 be distinct, fixed elements of $V$ (no numerical significance assumed). Let the maps $\phi_{2}, \ldots, \phi_{6}$ be defined so that, for all $x, y, w \in V$

$$
\begin{aligned}
\phi_{2}^{\wedge}(x) & =[\psi](2, x) \\
\phi_{3}^{\wedge}(x, y) & =[\psi](3,[\psi](0, x),[\psi](1, y)) \\
\phi_{4}^{\wedge}(x, y, w) & =[\psi](4,[\psi](0,[\psi](0, x),[\psi](1, y)),[\psi](1, w)) \\
\phi_{5}^{\wedge}(x) & =[\psi](5, x) \\
\phi_{6}^{\wedge}(x, y) & =[\psi](6,[\psi](0, x),[\psi](1, y)) .
\end{aligned}
$$

Note that all these functions are, perforce, in $F(I)$.

For $j=2, \ldots, 6$ define $V_{j}^{\wedge}=\operatorname{range}\left(\phi_{j}^{\wedge}\right)$. Utilizing the functions $\left[\mu_{i}\right], i=1,2,3$, of 5.9 , it is easy to show that the sets $V_{j}, j=2, \ldots, 6$ are computable in $I$, and, again by 5.9 , we can see that the functions $\phi_{j}^{\wedge}$ are one-to-one. Thus these sets and functions have the requisite properties. Let $\alpha^{\wedge}=[\psi](0)$ and $\psi^{\wedge}=[\psi](1)$. For $j=2, \ldots, 6$, let $\left[\varepsilon_{j}\right]$ denote the characteristic function of $V_{j}^{\wedge}$. Let $[\eta]$ be the characteristic function of the successor set $N$ of $\langle[s], z\rangle$, and let $\left[\mu_{i}\right], i=1,2,3$ be as in 5.9. Now pick a set $V_{1}^{\wedge}=\left\{s^{\wedge}, \varepsilon_{1}^{\wedge}, \ldots, \varepsilon_{6}^{\wedge}, \eta^{\wedge}, \mu_{1}^{\wedge}, \mu_{2}^{\wedge}, \mu_{3}^{\wedge}\right\}$ from the set $V-\left(\left\{\alpha^{\wedge}, \psi^{\wedge}\right\} \cup \bigcup_{j=2}^{6} V_{j}^{\wedge}\right)$ (that this set is nonempty, indeed infinite, follows from the definition of the $\left.V_{j}^{\wedge}\right)$. Then, where $\left[\varepsilon_{1}\right]$ is the characteristic function of $V_{1}^{\wedge}$, (note: $\left[\varepsilon_{1}\right] \in F(I)$ since $V_{1}^{\wedge}$ is finite), let $\phi_{1}^{\wedge}$ be the obvious map from $V_{1}^{\wedge}$, one-to-one onto the set of functions $\boldsymbol{F}^{\vee}=$ 
$\left\{[s],\left[\varepsilon_{1}\right],\left[\varepsilon_{2}\right], \ldots,\left[\varepsilon_{6}\right],[\eta],\left[\mu_{1}\right],\left[\mu_{2}\right],\left[\mu_{3}\right]\right\}$. This completes the specification of the 15-tuple $S^{\wedge}$. From 5.1 it follows that there is a corresponding U.R.S. $I^{\wedge}=\left\langle U, G^{\wedge}\right\rangle$ constructed from $S^{\wedge}$. Given $e \in V$, let $[e]^{\wedge}$ denote $G^{\wedge}(e)$.

That $I^{\wedge}$ is highly constructible is easy to see except for the existence of the requisite "inverses" $\left[\alpha_{1}^{\wedge-1}\right]^{\wedge}$ and $\left[\alpha_{2}^{\wedge-1}\right]^{\wedge}$ of $\left[\alpha^{\wedge}\right]_{2}^{\wedge}$. But for all $x, y \in V,\left[\alpha^{\wedge}\right]^{\wedge}(x, y)$ $=[\psi](6,[\psi](0, x),[\psi](1, y))$ and so since $\left[\mu_{i}\right]=\left[\mu_{i}^{\wedge}\right]^{\wedge} \in F\left(I^{\wedge}\right)$ for $i=1,2,3$, we have, by 2.3 , that there exist $\left[\alpha_{1}^{\wedge-1}\right]^{\wedge}$ and $\left[\alpha_{2}^{\wedge-1}\right]^{\wedge}$ in $F\left(I^{\wedge}\right)$ such that, for all $x \in V$,

$$
\begin{aligned}
& {\left[\alpha_{1}^{\wedge-1}\right]^{\wedge}(z)=\left[\mu_{1}\right]\left(0,\left[\mu_{2}\right](6, z)\right)} \\
& \left.\left[\alpha_{2}^{\wedge}\right]^{-1}\right]^{\wedge}(z)=\left[\mu_{1}\right]\left(1,\left[\mu_{3}\right](6, z)\right),
\end{aligned}
$$

which thus are the requisite "inverses" for $\left[\alpha^{\wedge}\right]_{2}^{\wedge}$.

It remains to show that $F\left(I^{\wedge}\right) \subset F(I)$. That $\left[\psi^{\wedge}\right]_{3}^{\wedge}=\phi_{4}^{\wedge} \in F(I)$ is clear from the definition of $\phi_{4}^{\wedge}$. Thus, by 6.1 , we need only show $F\left(1, I^{\wedge}\right) \subset F(I)$. To prove this, we employ 5.3. The first three conditions of 5.3 have been established above. Thus it remains only to establish the existence of the function $\left[t^{0}\right]_{2}$. Consider the function $t: V \times V \rightarrow U$ such that, for all $e, x \in V$,

$$
\begin{array}{rlrl}
t(e, x) & =\left(\phi_{1}^{\wedge}(e)\right)(x) & & \text { if } e \in V_{1}^{\wedge} \\
& =\phi_{2}^{\wedge}(x) & & \text { if } e=\psi^{\wedge}=[\psi](1) \\
& =\phi_{3}^{\wedge}\left(\left[\mu_{1}\right](2, e), x\right) & & \text { if } e \in V_{2} \\
& =\phi_{4}^{\wedge}\left(\left[\mu_{1}\right]\left(0,\left[\mu_{2}\right](3, e)\right),\left[\mu_{1}\right]\left(1,\left[\mu_{3}\right](3, e)\right), x\right) \text { if } e \in V_{3} \\
& =\left[\mu_{1}\right]\left(1,\left[\mu_{3}\right](4, e)\right) & & \text { if } e \in V_{4} \text { and } x=\left[\mu_{1}\right]\left(0,\left[\mu_{2}\right]\left(0,\left[\mu_{2}\right](4, e)\right)\right) \\
& =\left[\mu_{1}\right]\left(1,\left[\mu_{3}\right]\left(0,\left[\mu_{2}\right](4, e)\right)\right) & & \text { if } e \in V_{4} \text { and } x \neq\left[\mu_{1}\right]\left(0,\left[\mu_{2}\right]\left(0,\left[\mu_{2}\right](4, e)\right)\right) \\
& =\phi_{5}^{\wedge}(x) & & \text { if } e=\alpha^{\wedge}=[\psi](0) \\
& =\phi_{\hat{6}}^{\wedge}\left(\left[\mu_{1}\right](5, e), x\right) & & \text { if } e \in V_{5} \\
& =1 & & \text { if } e \notin \bigcup_{j=0}^{5} V_{j} .
\end{array}
$$

That this function is in $F(I)$ follows from the fact that the $\left[\varepsilon_{j}\right] \in F(I), j=1, \ldots, 5$; that the $\left[\mu_{i}\right] \in F(I), i=1,2,3$; that the $\phi_{j}^{\wedge} \in F(I), j=1, \ldots, 6$; and from 2.5. Thus, there exists $\left[t^{\circ}\right]_{2} \in F(I)$ such that $\left[t^{0}\right]_{2}=t$. That this is the desired function follows from inspection of the proof of 5.3 and the definitions of the $\phi_{j}^{\wedge}$ and $\mu_{i}$. Thus, $F\left(I^{\wedge}\right) \subset F(I)$ by 5.3 and 6.1 .

LEMMA 6.6. Every highly constructible U.R.S. $I=\langle U, G\rangle$ is functionally equivalent to a highly constructible U.R.S. in which $U-\bigcup_{i=0}^{6} V_{i} \neq \varnothing$.

Proof. Using 6.5 we shall construct a new, highly constructible U.R.S. $I^{\wedge}$ from sets $V_{0}^{\wedge}, V_{1}^{\wedge}, \ldots, V_{6}^{\wedge}$ such that $V-\bigcup_{i=0}^{6} V_{i}^{\wedge}$ is infinite (of Card $(V)$ ) and $F\left(I^{\wedge}\right)$ $\subseteq F(I)$. We will then show that there exists a *-total function $f$ such that $F\left(I_{f}^{\wedge}\right)=F(I)$ where $I_{f}^{\wedge}$ is the minimal $f$-extension of $I^{\wedge}$.

Since $I$ is highly constructible, we know that it contains a computable successor pair $\langle[s], z\rangle$ and that it contains $\psi \in V$ satisfying Axiom 3 and such that the sets 
range $\left([\psi]_{2}\right)$ and range $\left([\psi]_{3}\right)$ are computable in $I$. Hence, we can apply 6.5 and construct another highly constructible U.R.S. $I^{\wedge}$ such that $F\left(I^{\wedge}\right) \subset F(I)$. Now where $I^{\wedge}$ is constructed using $V_{0}^{\wedge}, V_{1}^{\wedge}, \ldots, V_{6}^{\wedge}$, we see, from the proof of 6.5 , that $V_{0}^{\wedge}$ and $V_{1}^{\wedge}$ are finite and

$$
\begin{aligned}
\bigcup_{i=0}^{6} V_{i}^{\wedge} \subseteq & \text { range }\left([[\psi](2)]_{1}\right) \cup \text { range }\left([[\psi](3)]_{2}\right) \\
& \cup \text { range }\left([[\psi](4)]_{2}\right) \cup \text { range }\left([[\psi](5)]_{1}\right) \\
& \cup \text { range }\left([[\psi](6)]_{2}\right) .
\end{aligned}
$$

But then, for every $a \notin V-\{2,3, \ldots, 6\}$, we know that range $\left([[\psi](a)]_{1}\right)$ is infinite and disjoint from $\bigcup_{i=2}^{6} V_{i}^{\wedge}$ (by 5.9) and hence $V-\bigcup_{i=0}^{6} V_{i}^{\wedge}$ must be infinite. Thus, it follows that we may form extensions of $I^{\wedge}$ in accordance with 6.4.

Let $\psi^{\wedge}=[\psi](1)$. Then $\left[\psi^{\wedge}\right]_{3}^{\wedge} \in F\left(I^{\wedge}\right) \subset F(I)$ and, in particular, there exist $p: V^{4} \rightarrow V, p \in F\left(I^{\wedge}\right) \subseteq F(I)$ such that for all $w, x, y, z \in V$,

$$
p(w, x, y, z)=\left[\psi^{\wedge}\right]^{\wedge}\left(0,\left[\psi^{\wedge}\right]^{\wedge}\left(0,\left[\psi^{\wedge}\right]^{\wedge}(0, w, x), y\right), z\right) .
$$

Furthermore, $p$ is one-to-one and range $(p)$ is computable in $I^{\wedge}$ (and thus in $I$ ) since $I^{\wedge}$ is highly constructible.

Let $[U]_{3}$ and $[T]_{3}$ be the functions employed in the normal form Theorem 5.5 for $I$. Let $f$ be the function such that, for all $v \in V$,

$$
\begin{aligned}
f(v) & =[U]_{3}(x, y, z) & & \text { if } v=p(0, x, y, z) \\
& =[T]_{3}(x, y, z) & & \text { if } v=p(1, x, y, z) \\
& =0 & & \text { otherwise. }
\end{aligned}
$$

Clearly, $f \in F(I)$ and any U.R.S. which contains both $p$ and $f$ contains $[U]_{3}$ and $[T]_{3}$. Thus, in particular, the $f$-extension $I_{f}^{\wedge}$ of $I^{\wedge}$ (given by 6.4) contains $[U]_{3}$ and $[T]_{3}$ and thus, $F(1, I) \subseteq F\left(I_{f}^{\wedge}\right)$. But then $F(I) \subseteq F\left(I_{f}^{\wedge}\right)$ by 6.1 using $\left[\psi^{\wedge}\right]_{3}^{\wedge}$. Conversely, $F\left(I_{f}^{\wedge}\right) \subset F(I)$ since $F\left(I^{\wedge}\right) \cup\{f\} \in F(I)$ and $I_{f}^{\wedge}$ is the minimal $f$-extension of $I^{\wedge}$. Thus, $F\left(I_{f}^{\wedge}\right)=F(I)$. But $I_{f}^{\wedge}$ is constructed using the sets $V_{0}^{\wedge}, V_{1}^{\wedge} \cup\left\{v_{f}\right\}, V_{2}^{\wedge}, \ldots, V_{6}^{\wedge}$ where the $V_{i}^{\wedge}, i=0, \ldots, 6$, are the sets used to define $I^{\wedge}$, thus, the desired result follows from the above proof that $V-\bigcup_{i=0}^{6} V_{i}^{\wedge}$ is infinite.

THEOREM 6.7. Let I be a highly constructible U.R.S. and let $f: U \rightarrow U$ be a *admissible function. Then there exists a minimal f-extension $I_{f}$ of $I$ in the sense that if $I^{\prime}$ is any f-extension of $I$, then $F\left(I_{f}\right) \subset F\left(I^{\prime}\right)$.

Proof. This result is an immediate corollary of 6.4 and 6.6.

The above result shows that given a highly constructible U.R.S. $I=\langle U, G\rangle$, we may associate every $*$-admissible function $f: U \rightarrow U$ with a minimal $f$-extension of I. We now prove the converse, that every extension of a highly constructible U.R.S. $I=\langle U, G\rangle$ is a minimal $f$-extension of $I$ for some $*$-admissible $f: U \rightarrow U$. 
THEOREM 6.8. Let $I=\langle U, G\rangle$ be a highly constructible U.R.S. and let $I^{\prime}=\left\langle U, G^{\prime}\right\rangle$ be an extension of $I$. Then there exists $f \in F\left(I^{\prime}\right)$ such that $I^{\prime}$ is functionally equivalent to the minimal f-extension $I_{f}$ of $I$.

Proof. Given $u \in U$, let $[u]$ denote $G(u)$ and let $[u]^{\prime}$ denote $G^{\prime}(u)$.

Let $\left[i^{\prime}\right]^{\prime}$ denote the identity function in $F\left(I^{\prime}\right)$ and let $[\psi]$ denote the function given by Axiom 3 for $I$. Given a fixed element $c$ of $V$, we know there exists $t \in V$ such that, for all $x, y \in V,[t](x, y)=[\psi](c, x, y)$. Since $I$ is highly constructible, we know range $\left([\psi]_{3}\right)$ is computable and thus that range $\left([t]_{2}\right)$ is computable. Taking $\left[\lambda_{1}\right]$ and $\left[\lambda_{2}\right]$ as in 3.5, we see, furthermore, that for all $x, y \in V$,

$$
\left[\lambda_{1}\right]([t](x, y))=x, \quad\left[\lambda_{2}\right]([t](x, y))=y .
$$

Since $I^{\prime}$ is an extension of $I$, we know that all these functions are in $F\left(I^{\prime}\right)$. It follows easily then from 2.5 that there exists $[\rho]_{1}^{\prime} \in F\left(I^{\prime}\right)$ such that, for every $w \in V$,

$$
\begin{aligned}
{[\rho]^{\prime}(w) } & =\left[i^{\prime}\right]^{\prime}(x, y)=[x]^{\prime}(y) & & \text { if } w=[t](x, y) \\
& =0 & & \text { otherwise. }
\end{aligned}
$$

Now take $f=[\rho]_{1}^{\prime}$ and consider the minimal $f$-extension $I_{f}=\left\langle U, G_{f}\right\rangle$ of $I$. Given $u \in U$, let $[u]^{f}$ denote $G_{f}(u)$. Since $F(I) \cup\left\{f=[\rho]_{1}^{\prime}\right\} \subseteq F\left(I_{f}\right), F\left(I^{\prime}\right)$ and $I_{f}$ is minimal, it follows that $F\left(I_{f}\right) \subseteq F\left(I^{\prime}\right)$. On the other hand, since $f=[\rho]_{1}^{\prime}$ and $[t]_{2}$ are in $F\left(I_{f}\right)$, it follows that $\left[i^{\prime}\right]_{2}^{\prime}=[\rho]_{1}^{\prime} \circ[t]_{2}$ is in $F\left(I_{f}\right)$ and so, since $\left[i^{\prime}\right]^{\prime}(x, y)=[x]^{\prime}(y)$ for all $x, y \in V$, we have $F\left(1, I^{\prime}\right) \subseteq F\left(1, I_{f}\right)$. But then by $6.1, F\left(I^{\prime}\right) \subseteq F\left(I_{f}\right)$ and so $F\left(I_{f}\right)$ $=F\left(I^{\prime}\right)$.

THEOREM 6.9. Let $I^{\wedge}$ and I be highly constructible U.R.S. Then I is an extension of $I^{\wedge}$ if and only if there exists $a *$-total function $f$ such that $I$ is the minimal f-extension of $I^{\wedge}$.

Proof. By the same argument as used in the second half of the proof of 6.6.

From the above result and 5.4, it follows that if we take $I$ to be the U.R.S. of partial recursive functions, then its highly constructible extensions are precisely the set of U.R.S. corresponding to the sets of functions recursive in total functions. To put it another way, if $f$ is a total function on $N$, then the set of functions recursive in $f$ is precisely the function set of the minimal $f$-extension of $I$, and if $I^{\prime}$ is a highly constructible extension of $I$, then $F\left(I^{\prime}\right)$ is the set of functions recursive in $f$ for some total function $f$.

This result leads us naturally to consider the $f$-extensions of a U.R.S. $I$ where $f$ is not $*$-total. In the next three results, we show that there exist extensions which are not highly constructible (i.e., in which $f$ cannot be replaced by a $*$-total function). As a corollary of these results, we show that there exist U.R.S. in which not all semicomputable sets are recursively enumerable.

THEOREM 6.10. Let I be a highly constructible U.R.S. and let $f$ and $h$ be 1-ary *-admissible functions such that $h$ is *-total and for every $x \in V, f(x) \neq *$ implies 
$f(x)=h(x)$. Then where $I_{f}=\left\langle U, G_{f}\right\rangle$ and $I_{h}=\left\langle U, G_{h}\right\rangle$ are respectively the minimal $f$-and minimal h-extensions of $I$, we have that every *-total function in $F\left(I_{f}\right)$ is also in $F\left(I_{h}\right)$.

Proof. We shall first prove that every 1-ary *-total function in $F\left(I_{f}\right)$ is in $F\left(I_{h}\right)$.

Let $I$ be constructed from $S=\left\langle F, \alpha, \psi, V_{1}, \ldots, V_{6}, \phi_{1}, \ldots, \phi_{6}\right\rangle$ and let $I_{f}$ and $I_{h}$ be constructed from $S^{f}=\left\langle\boldsymbol{F}^{f}, \alpha, \psi, V_{1}^{f}, V_{2}, \ldots, V_{6}, \phi_{1}^{f}, \phi_{2}, \ldots, \phi_{6}\right\rangle$ and $S^{h}=\left\langle F^{h}, \alpha, \psi\right.$, $\left.V_{1}^{h}, V_{2}, \ldots, V_{6}, \phi_{1}^{h}, \phi_{2}, \ldots, \phi_{6}\right\rangle$ respectively, where $\boldsymbol{F}^{f}=\boldsymbol{F} \cup\{f\}, \boldsymbol{F}^{h}=\boldsymbol{F} \cup\{h\}, V_{1}^{f}=V_{1}^{h}$ $=V_{1} \cup\{v\} ; v \in V=V-\bigcup_{i=0}^{6} V_{i} ; \phi_{1}^{f}=\phi_{1} \cup\{\langle v, f\rangle\}, \phi_{1}^{h}=\phi_{1} \cup\{\langle v, h\rangle\}$. Let $\Lambda_{n}^{f}$ and $g_{n}^{f}$ denote the sets and functions used to construct $I_{f}$, and let $\Lambda_{n}^{h}$ and $g_{n}^{h}$ denote the sets and functions used to construt $I_{h}$. For all $e \in V$, let $[e]^{f}$ denote $G_{f}(e)$ and let $[e]^{h}$ denote $G_{h}(e)$.

We claim now that for all integers $n \geqq 0, \Lambda_{n}^{f} \subseteq \Lambda_{n}^{h}, \Lambda_{n+1}^{f}-\Lambda_{r}^{f} \subseteq \Lambda_{n+1}^{h}-\Lambda_{n}^{h}$, and, if $\langle e, x\rangle \in \Lambda_{n}^{f}$, then $g_{n}^{f}(e, x)=g_{n}^{h}(e, x)$. The desired result, that $[e]^{f}(x) \neq *$ implies $[e]^{\prime}(x)=[e]^{h}(x)$ will then follow immediately from the construction in the proof of 5.1 .

We proceed by induction on $n$. For $n=0$, the result is immediate and, indeed, $\Lambda_{0}^{f}=\Lambda_{0}^{h}$. Assume now that it has been proved up to $n=k \geqq 0$, and consider the case for $k+1$.

Say $\langle e, x\rangle \in \Lambda_{k+1}^{f}$. Then, by definition, we have $\left(\langle e, x\rangle \in \Lambda_{k}^{f}\right)$ or $\left(\left(\langle e, x\rangle \notin \Lambda_{k}^{f}\right)\right.$ and $\left(e \in V_{6}\right)$ and $\left(\left\langle\left[\alpha_{1}^{-1}\right](e), x\right\rangle \in \Lambda_{k}^{f}\right)$ and $\left(\left\langle\left[\alpha_{2}^{-1}\right](e), x\right\rangle \in \Lambda_{k}^{f}\right)$ and $\left(\left\langle g_{k}^{f}\left(\left[\alpha_{1}^{-1}\right](e), x\right)\right.\right.$, $\left.\left.\left.g_{k}^{f}\left(\left[\alpha_{2}^{-1}\right](e), x\right)\right\rangle \in \Lambda_{k}^{f}\right)\right)$. But then, by the induction hypothesis, it follows that the same statement holds with $g_{k}^{h}$ replacing $g_{k}^{f}$, and $\Lambda_{k}^{h}$ replacing $\Lambda_{k}^{f}$ and so, $\langle e, x\rangle$ $\in \Lambda_{k+1}^{f}$. Thus, $\Lambda_{k+1}^{f} \subseteq \Lambda_{k+1}^{h}$.

To see that $\Lambda_{k+1}^{f}-\Lambda_{k}^{f} \subseteq \Lambda_{k+1}^{h}-\Lambda_{k}^{h}$, assume there exists $\langle e, x\rangle \in\left(\left(\Lambda_{k+1}^{f}-\Lambda_{k}^{f}\right)\right.$ $\left.-\left(\Lambda_{k+1}^{h}-\Lambda_{k}^{h}\right)\right)$. Since $\Lambda_{k+1}^{f} \subseteq \Lambda_{k+1}^{h}$, it follows that $\langle e, x\rangle \in \Lambda_{k}^{h}$, but $\langle e, x\rangle \notin \Lambda_{k}^{f}$. Since $\Lambda_{0}^{f}=\Lambda_{0}^{h}$, it follows that $k>0$ and $\langle e, x\rangle \notin \Lambda_{0}^{h}$; yet, $e \in V_{6}$. Now since $\langle e, x\rangle \in \Lambda_{k+1}^{f}-\Lambda_{k}^{f}$, we know that the pairs $\left\langle\left[\alpha_{1}^{-1}\right](e), x\right\rangle,\left\langle\left[\alpha_{2}^{-1}\right](e), x\right\rangle$ and $\left\langle g_{k}^{f}\left(\left[\alpha_{1}^{-1}\right](e), x\right), g_{k}^{f}\left(\left[\alpha_{2}^{-1}\right](e), x\right)\right\rangle$ are in $\Lambda_{k}^{f}$ and thus, by the induction hypothesis, in $\Lambda_{k}^{h}$. But clearly, these three pairs cannot all be in $\Lambda_{k-1}^{f}$ since this would imply $\langle e, x\rangle \in \Lambda_{k}^{f}$. But $\langle e, x\rangle \in \Lambda_{k}^{h},\langle e, x\rangle \notin \Lambda_{0}^{h}$ implies that all these pairs are in $\Lambda_{k-1}^{h}$. It follows then that at least one of these pairs is in $\Lambda_{k}^{f}-\Lambda_{k-1}^{f}$, but not in $\Lambda_{k}^{h}-\Lambda_{k-1}^{h}$; i.e., $\Lambda_{k}^{f}-\Lambda_{k-1}^{f} \nsubseteq \Lambda_{k}^{h}-\Lambda_{k-1}^{h}$, which contradicts the induction hypothesis. Thus, it must be that $\Lambda_{k+1}^{f}-\Lambda_{k}^{f} \subseteq \Lambda_{k+1}^{h}-\Lambda_{k}^{h}$.

Now, from this last result, the definition of $g_{k+1}^{f}$ and $\Lambda_{k+1}^{f}$ and the induction hypothesis, we now have that, for all $\langle e, x\rangle \in \Lambda_{k+1}^{f}$

$$
\begin{aligned}
g_{k+1}^{f}(e, x) & = \begin{cases}g_{k}^{f}(e, x)=g_{k}^{h}(e, x) & \text { if }\langle e, x\rangle \in \Lambda_{k}^{f} \subseteq \Lambda_{k}^{h} \\
g_{k}^{f}\left(g_{k}^{f}\left(\left[\alpha_{1}^{-1}\right](e), x\right), g_{k}^{f}\left(\left[\alpha_{2}^{-1}\right](e), x\right)\right)=g_{k}^{h}\left(g_{k}^{h}\left(\left[\alpha_{1}^{-1}\right](e), x\right), g_{k}^{h}\left(\left[\alpha_{2}^{-1}\right](e), x\right)\right) & \text { if }\langle e, x\rangle \in \Lambda_{k+1}^{f}-\Lambda_{k}^{f} \subseteq \Lambda_{k+1}^{h}-\Lambda_{k}^{h}\end{cases} \\
= & g_{k+1}^{h}(e, x) .
\end{aligned}
$$

This completes the inductive step and so the claims are proved by induction for all $n$. But then, since $\langle e, x\rangle \in \Lambda_{n}^{f}$ implies $[e]^{f}(x)=g_{n}^{f}(e, x)$ and $\langle e, x\rangle \notin \bigcup_{n=0}^{\infty} \Lambda_{n}^{f}$ 
implies $[e]^{f}(x)=*$ (see 5.1), it follows immediately that $F\left(I_{f}\right)$ and $F\left(I_{h}\right)$ contain the same 1-ary $*$-total functions.

To see that $F\left(I_{f}\right)$ and $F\left(I_{h}\right)$ contain the same $n$-ary $*$-total functions for $n>1$, we proceed as follows: Say that $F\left(I_{f}\right)$ contains an $n$-ary *-total function $[g]_{n}^{f}$ which is not in $F\left(I_{h}\right)$. By 5.9 we have a function $\left[p_{n}\right] \in F(I)$ by means of which we may (reversibly) encode $n$-tuples of elements of $V$ as single elements. But then, since $F(I) \subseteq F\left(I_{f}\right), F\left(I_{h}\right)$, it is clear that there exists a function $\left[g^{\prime}\right] \in F\left(I_{f}\right)$ such that, for all $x \in V$,

$$
\begin{array}{rlrl}
{\left[g^{\prime}\right]^{f}(x)} & =[g]^{f}\left(y^{(n)}\right) & & \text { if } x=\left[p_{n}\right]\left(y^{(n)}\right) \text { for some } y^{(n)} \in V^{n} \\
& =[g]^{f}(x, x, \ldots, x) & & \text { otherwise. } \\
n \text { times } & &
\end{array}
$$

But then, since $\left[g^{\prime}\right]_{1}^{f}$ is clearly $*$-total, we have $\left[g^{\prime}\right]_{1}^{f} \in F\left(I_{h}\right)$ by the first part of the proof, and this implies that $[g]^{f}=\left[g^{\prime}\right]^{f} \circ\left[p_{n}\right]_{n} \in F\left(I_{h}\right)$, contradicting the assumption that $[g] \notin F\left(I_{h}\right)$. Thus every $*$-total function in $\grave{F}\left(I_{f}\right)$ must be in $F\left(I_{h}\right)$.

COROLLARY 6.11. If I is a highly constructible U.R.S. and fis a 1-ary *-admissible function and there exists a *-total function $[h]_{1} \in F(I)$ such that, for every $x \in V$, $f(x) \neq *$ implies $[h](x)=f(x)$, then every $*$-total function in the minimal $f$-extension of $I$ is already in $F(I)$. Thus, in particular, if $p$ is the semicharacteristic function of any set $A \subset V$ (or of any 1-ary predicate on $V$ ), then $F\left(I_{p}\right)$ does not contain any *-total functions not already in $F(I)$ (take $[h]_{1}=\left[k_{0}\right]$ as in 2.1).

Proof. Left to the reader.

THEOREM 6.12. If I is a highly constructible U.R.S. then it has an extension I' which is not highly constructible.

Proof. Given the highly constructible U.R.S. $I$, let $[u]$ denote $G(u)$ for all $u \in U$. Now let $p$ be the semicharacteristic function of the predicate $P(x) \Leftrightarrow[x](x)=*$, and let $I^{\prime}=I_{p}$, the minimal $p$-extension of $I$.

Now the predicate $P(x)$ is semicomputable in $I^{\prime}$ since $p \in F\left(I^{\prime}\right)$; however, $P(x)$ is not semicomputable in $I$ for, supposing that it were, then there would exist $\left[u_{p}\right] \in F(I)$ such that $\left[u_{p}\right]=p$. But then we would have

$$
\begin{array}{rlrl}
{\left[u_{p}\right]\left(u_{p}\right)=p\left(u_{p}\right)=0} & & \text { if }\left[u_{p}\right]\left(u_{p}\right)=* \\
& =* & & \text { if }\left[u_{p}\right]\left(u_{p}\right)=0,
\end{array}
$$

a contradiction. From this, it also follows by 4.2 that $P(x)$ is not computable in $I$ (see 4.2). On the other hand, the predicate $\sim P(x) \Leftrightarrow[x](x) \neq *$ is semicomputable in $I$ (with semicharacteristic function $\left[k_{0}\right]([x](x))$ ). But then, since $F(I) \subset F\left(I^{\prime}\right)$, it follows that both $P(x)$ and $\sim P(x)$ are semicomputable in $I^{\prime}$, and, since, by 6.11 , every *-total function in $F\left(I^{\prime}\right)$ is also in $F(I)$, it follows that $P(x)$ is not computable in $I^{\prime}$. But if $I^{\prime}$ was highly constructible, then, by 5.6, $P(x)$ and $\sim P(x)$ semicomputable in $I^{\prime}$ would imply $P(x)$ computable in $I^{\prime}$. Thus, $I^{\prime}$ cannot be highly constructible. 
THEOREM 6.13. Let a set $A \subset V$ be said to be recursively enumerable in a U.R.S.I if there exist a $*$-total function $[f]_{n} \in F(I)$ such that $A=$ range $\left([f]_{n}\right)$. There exists a U.R.S. $I^{\prime}$ and a set $A$ such that $A$ is semicomputable in $I^{\prime}$ but $A$ is not recursively enumerable in $I^{\prime}$.

Proof. Let $I$ be the highly constructible U.R.S. of partial recursive functions. Let $p$ be the semicharacteristic function of the set $A=\{x \mid[x](x)=*\}$. By the argument given in the preceding theorem, we know that $p \notin F(I)$ and that, since all recursively enumerable sets in the partial recursive functions are semicomputable sets, $A$ is not recursively enumerable in $I$. Now let $I^{\prime}=I_{p}$ be the minimal $p$-extension of $I$. Then $p \in F\left(I^{\prime}\right)$ so $A$ is semicomputable in $I$. But if $A$ is recursively enumerable in $I^{\prime}$, there exists a total function $[f]_{n}^{\prime}$ in $F\left(I^{\prime}\right)$ such that range $\left([f]_{n}^{\prime}\right)=A$, but then, by $6.11,[f]_{n}^{\prime}$ is also in $I$ and so $A$ is recursively enumerable in $I$, which we know it is not. Thus $A$ is semicomputable in $I^{\prime}$, but not recursively enumerable in $I^{\prime}$.

It was pointed out by a referee that the above result follows directly from the fact that the $\Pi_{1}^{1}$-functions form a U.R.S. since the "recursively enumerable" $\Pi_{1}^{1}$ sets are precisely the hyperarithmetic sets which are all "computable" in our sense, yet the set $\{x \mid[x](x) \neq x\}$ is clearly semicomputable, but not computable.

To complete the paper, we show that the extension relation induces an upper semilattice on the set of extensions of a highly constructible U.R.S. and on the set of all $*$-admissible functions.

LEMMA 6.14. Given a highly constructible U.R.S. I, a minimal $g^{\prime}$-extension $I_{g^{\prime}}$ of $I$, and a minimal $g^{\prime \prime}$-extension $I_{g^{\prime \prime}}$ of $I$, then there exists a function $g^{\wedge}$ and a corresponding minimal $g^{\wedge}$-extension $I_{g^{\wedge}}$ of $I$ such that $I_{g^{\wedge}}$ is also the minimal $\left\{g^{\prime}, g^{\prime \prime}\right\}$-extension of $I$.

Proof. Given $g^{\prime}, g^{\prime \prime}$, let $g^{\wedge}$ be such that where $c^{\prime}, c^{\prime \prime} \in V, c^{\prime} \neq c^{\prime \prime}$,

$$
\begin{aligned}
g^{\wedge}(x) & =g^{\prime}(y) & & \text { if } x=[\psi]\left(c^{\prime}, y\right) \\
& =g^{\prime \prime}(y) & & \text { if } x=[\psi]\left(c^{\prime \prime}, y\right) \\
& =* & & \text { otherwise. }
\end{aligned}
$$

We see then that, for every $x \in V, g^{\prime}(x)=g^{\wedge}([[\psi](c)](x))$ and so $g^{\prime} \in F\left(I_{g^{\wedge}}\right)$, and similarly, $g^{\prime \prime} \in F\left(I_{g^{\wedge}}\right)$. Thus, $I_{g^{\wedge}}$ is a $\left\{g^{\prime}, g^{\prime \prime}\right\}$-extension of $I$. On the other hand, let $I^{\wedge}$ be any $\left\{g^{\prime}, g^{\prime \prime}\right\}$-extension of $I$. Then since the predicates $x=[\psi](c, y)$ and $x=[\psi]\left(c^{\prime}, y\right)$ are computable in $I$ by virtue of it being highly constructible, it follows that $g^{\wedge} \in F\left(I^{\wedge}\right)$, and so $F\left(I_{g^{\wedge}}\right) \subseteq F\left(I^{\wedge}\right)$, so $I_{g^{\wedge}}$ must be the minimal $\left\{g^{\prime}, g^{\prime \prime}\right\}$-extension of $I$.

COROLlaRY 6.15. Let $I=\langle U, G\rangle$ be a highly constructible U.R.S. and let $\mathscr{T}$ denote the set of all $*$-admissible functions on $U$ partitioned in accordance with the equivalence relation $\equiv$ where $f \equiv g$ if and only if $F\left(I_{f}\right)=F\left(I_{g}\right)$. Then $\mathscr{F}$ is an upper semilattice under the partial ordering < where, if $\mathscr{F}(f)$ and $\mathscr{F}(g)$ are elements of $\mathscr{F}$ (with respective representatives $f$ and $g)$, then $\mathscr{F}(f)<\mathscr{F}(g)$ if and only if $F\left(I_{f}\right) \subset F\left(I_{g}\right)$.

Proof. Follows immediately from 6.7, 6.8, and 6.14. 


\section{REFERENCES}

1. M. Davis, Computability and unsolvability, McGraw-Hill, New York, 1958.

2. R. M. Friedberg, Three theorems on recursive enumeration. I. Decomposition. II. Maximal set. III. Enumeration without duplication, J. Symbolic Logic 23 (1958); 309-316.

3. H. M. Friedman, Personal communication.

4. H. Rogers, Jr., Gödel numberings of partial recursive functions, J. Symbolic Logic 23 (1958), 331-341.

5. - Theory of recursive functions and effective computability, McGraw-Hill, New York, 1967.

6. M. Schönfinkel, Über die Bausteine der Mathematischen Logik, Math. Ann. 92 (1922), 305-316.

7. R. M. Smullyan, Theory of formal systems, Ann. of Math. Studies, No. 47, Princeton Univ. Press, Princeton, N. J., 1961.

8. H. R. Strong, Jr., An algebraic approach through uniformly reflexive structures to generalized recursive function theory, Doctoral Dissertation, Univ. of Washington, Seattle, Wash., 1967.

9. —_ Algebraically generalized recursive function theory, IBM J. Res. Develop. 12. (1968), 465-475.

10. E. G. Wagner, Uniformly reflexive structures: Toward an abstract theory of computability, Doctoral Dissertation, Columbia Univ., New York, 1963. Also, IBM Res. Rep. RC 934, 1963.

11. —_, Uniformly reflexive structures. II, IBM Res. Rep. RC 1135, 1964.

12. - Uniformly reflexive structures. III; Constructible and highly constructible U.R.S., IBM Res. Rep. RC 1341, 1964.

13. - Uniformly reflexive structures. IV; Many-one reducibility and strongly creative functions, IBM Res. Rep. RC 1397, 1965.

IBM Watson Research CENTER, YorkTOWN HeIGHTS, New YoRK 